\title{
8 Steuerung und Gestaltung von räumlichen Schnittstellen der Mobilität
}

Betrachtungen angesichts des automatisierten Fahrens

Emilia M. Bruck, Rudolf Scheuvens, Martin Berger

1. Einleitung

2. Auswirkungen des automatisierten Fahrens auf öffentliche Räume 135

$\begin{array}{lll}2.1 & \text { Problemstellung } & 135\end{array}$

$\begin{array}{lll}2.2 & \text { Betrachtungsfokus } & 137\end{array}$

3. Strategische Rahmenbedingungen räumlicher Schnittstellen 137

3.1 Bewertungen integrieren: Einsatzgebiete, Modi und zulässige Geschwindigkeiten 138

3.2 Stationsnetze erweitern: Ausbau um Haltezonen und Sammelpunkte 140

3.3 Zentralitäten stärken: Verknüpfung von Nutzungen, Wegen und Zielen 143

3.4 Adaptivität sichern: Offenheit für zukünftigen Nutzungswandel 145

4. Kleinräumige Gestaltung multimodaler öffentlicher Räume 146

4.1 Multimodale öffentliche Räume: Entflechtung und Bündelung von Modi 147

4.2 Flexible Flächendimensionierung: bedarfsabgestimmt oder tageszeitabhängig 149

4.3 Sanfte Übergänge: durchlässig und zum Verweilen einladend 150

4.4 Ausstattung und Adressbildung: im Wechselspiel mit dem Umfeld 152

$\begin{array}{lr}\text { 5. Resümee und Ausblick } & 153\end{array}$

$\begin{array}{lr}\text { Literatur } & 154\end{array}$

Emilia M. Bruck

TU Wien, future.lab Research Center und Forschungsbereich Örtliche Raumplanung (IFOER) emilia.bruck@tuwien.ac.at

Rudolf Scheuvens

TU Wien, future.lab Research Center und Forschungsbereich Örtliche Raumplanung (IFOER) rudolf.scheuvens@tuwien.ac.at

Martin Berger

TU Wien, Forschungsbereich Verkehrssystemplanung (IVS)

martin.kp.berger@tuwien.ac.at 


\section{EINLEITUNG}

Der vorliegende Beitrag widmet sich den Veränderungen im öffentlichen Raum, die mit der digitalen Vernetzung und Automatisierung im Mobilitätswesen einhergehen. Hierzu werden speziell räumliche Schnittstellen der Mobilität in den Fokus gerückt. Gemeint sind dabei Räume der Interaktion, der Verknüpfung unterschiedlicher Modi und des Übergangs im öffentlichen Raum. Aufgrund der zunehmenden Vielfalt an Mobilitätsangeboten und neuen Nutzungsanforderungen gewinnt schon heute die Gestaltung von räumlichen Schnittstellen an planerischer Bedeutung. Zumal sich Orte des Zugriffs auf plattformbasierte Mobilitätsangebote nicht selten als Brennpunkte in öffentlichen Räumen abzeichnen. Im Hinblick auf eine mögliche Einführung selbstfahrender Fahrzeuge ist anzunehmen, dass sich die stadtbildprägende Relevanz von räumlichen Schnittstellen als Schwellen zwischen Verkehrsraum und Fußweg sowie Bewegung und Aufenthalt verschärfen wird: zum einen, da die Einführung automatisierter Mobilität zu einem Rückgang des privaten Automobilbesitzes und der Verbreitung von Sharing-Angeboten beitragen könnte (Zhang et al. 2015), zum anderen, da die Annahme besteht, dass automatisierte Fahrzeuge nicht zwingend an ihrem Zielort parken, sondern eigenständig in eine entlegene Parkgarage fahren können (Zhang/Wang 2020). In beiden Fällen würde der Bedarf nach Parkplatzflächen im öffentlichen Raum sinken, währenddessen Möglichkeiten des Zu- und Ausstiegs, des Umstiegs auf andere Modi und des Zulieferns von Waren an Nachfrage gewinnen würden.

Vor diesem Hintergrund gilt es, über die rein funktionale Lösung der räumlich-physischen Vernetzung hinaus zu denken und die Wechselwirkungen mit dem jeweiligen Kontext - Stadtgestalt, Nutzungsnachbarschaft, Verkehrsaufkommen und Zugänglichkeit - in ganzheitlichen Planungsansätzen zu berücksichtigen. Mögliche Gestaltungsansätze umfassen die Ausweisung von Haltezonen und Sammelpunkten im öffentlichen Raum, die Erweiterung bestehender Haltestellen und Bahnhöfe oder auch die Anreicherung von Mobilitätspunkten mit Versorgungsfunktionen, die dann als kleinräumige Zentren an Bedeutung gewinnen können.

Auch wenn derzeit unklar ist, ob und unter welchen Umständen automatisierte Fahrzeuge weitreichend zum Einsatz kommen werden, ist es aus Sicht der Stadtentwicklung entscheidend, dass die automatisierten Regelwerke der Zukunft auf Basis der räumlichen Steuerungs- und Nutzungsweisen der Übergangsphase verfasst werden (Marsden et al. 2020). Anstatt tradierte Planungsansätze, wonach das Automobil das Mobilitätssystem und den öffentlichen Raum dominiert, durch automatisierte Systeme reproduzieren zu lassen, gilt es frühzeitig zu ergründen, inwieweit diese mit der Einführung neuer Mobilitätsformen zu überwinden sind. Die Auseinandersetzung mit kleinräumigen Veränderungen unter den Vorzeichen automatisierter Mobilität wirft demnach nicht nur Fragen nach der künftigen Verknüpfung und Interaktion unterschiedlicher Modi auf, sondern auch nach der Nutzungsvielfalt im öffentlichen Raum, dem fließenden Übergang zu umliegenden Funktionen sowie nach der Erneuerung urbaner Qualitäten. Nicht zuletzt ist zu hinterfragen, welche Folgen die Anwendung neuer Mobilitätslösungen für die Zielsetzung hat, hochwertige öffentliche Räume umzusetzen, die sich immer auch durch das Ungeplante, das Unvorhergesehene auszeichnen. Das Ziel der AutorInnen ist es, einen praxisnahen Beitrag zur Diskussion um angemessene Formen des stadt- und mobilitätsplanerischen Handelns im Kontext automatisierter Mobilität zu leisten. Dazu werden zunächst die ambivalenten Wirkungsweisen neuer Mobilitätstechnologien erläutert und in Folge acht planungsrelevante Betrachtungen vorgestellt, die zwei Handlungsebenen zugeordnet sind:

- strategische Rahmenbedingungen räumlicher Schnittstellen und

- kleinräumige Gestaltung multimodaler öffentlicher Räume. 
Der vorliegende Beitrag ist auch als ein Plädoyer dafür zu lesen, dass sich unter den Vorzeichen des automatisierten Fahrens der Status öffentlicher Räume als umkämpftes Terrain verschärft und es demnach einer achtsamen Justierung von Planungsansätzen bedarf, um die urbanen Qualitäten eines Ortes zu wahren (Schmid 2016). Dazu wird in den Betrachtungen die Relevanz von integrierten Ansätzen unterstrichen, um angesichts neuer Mobilitätsangebote und selbstfahrender Fahrzeuge intermodale Wege und aktive Mobilität zu fördern - zumal die räumliche Gestaltung eine maßgebliche externe Einflussgröße für die Verkehrsmittelwahl darstellt (Konrad/Groth 2019).

\section{AUSWIRKUNGEN DES AUTOMATISIERTEN FAHRENS AUF ÖFFENTLICHE RÄUME}

\section{$2.1 \quad$ PROBLEMSTELLUNG}

Aufgrund der digitalen Vernetzung und Automatisierung sind städtische und regionale Mobilitätssysteme in einem schleichenden Wandel begriffen. Als Vorboten automatisierter Verkehre gelten speziell stationsungebundene Mitfahrdienste (wie z. B. Uber oder Lyft), die sich im Laufe der vergangenen Jahre auch in europäischen Groß- und Mittelstädten ausgebreitet haben (Soike et al. 2019, Erhardt et al. 2019). Durch die Komfortvorteile der Tür-zu-Tür-Beförderung bestechen diese Angebote gegenüber dem herkömmlichen öffentlichen Personennahverkehr (ÖPNV) besonders in Räumen hoher Siedlungs- und Nachfragedichte. Planerisch relevante Faktoren wie die Reisezeit oder Flächeneffizienz des Systems treten in den Hintergrund, währenddessen die Flexibilität, Verfügbarkeit und individuelle Anpassungsfähigkeit des Angebots an Bedeutung gewinnen.

Mit hochautomatisierten Fahrzeugen (SAE-Level 4 nach SAE International 2018), so verspricht es die Industrie, könnte das Fahrerlebnis weiter an Komfort, Sicherheit und Effizienz gewinnen. Das automatisierte Gefahrenwerden soll einerseits jenen zugutekommen, die konventionelle Privatfahrzeuge selbst nicht steuern können, d. h. Mobilitätseingeschränkten, Kindern und der älteren Bevölkerung (Gavanas 2019). Andererseits soll mit der Automatisierung öffentlicher Angebote und privater Mobilitätsdienste die Verkehrsmittelwahl jener erweitert werden, die in autoabhängigen Gebieten leben oder sich das individuelle Automobil nicht leisten können. Demnach besteht besonders für suburbane und ländlich-periphere Räume die Hoffnung, infolge von Einsparungen bei Betriebs- und Personalkosten eine wirtschaftlich rentable Versorgung mittels selbstfahrender Zubringerdienste, Ortsbusse oder Dorfverbindungen sichern zu können (vgl. Jürgens 2020: 40).

Inzwischen zeigt sich in nordamerikanischen Innenstädten, dass mit einem weitreichenden Umstieg auf Mitfahrdienste das Verkehrsaufkommen drastisch zunehmen und ungeregeltes Halten entlang der Bordsteinkante zu Nutzungskonflikten mit dem ÖPNV und Radfahrenden führen kann (Schaller 2017, Erhardt et al. 2019). Der einhergehende Anstieg von Zu- und Ausstiegssituationen ist beispielhaft für eine Intensivierung und Diversifizierung von Nutzungsansprüchen im öffentlichen Raum (Marsden et al. 2020), die mit dem Aufkommen von Lieferdiensten, Angeboten der Mikromobilität und plattformbasierten Mitfahrdiensten einhergehen. Zudem entstehen teils widersprüchliche Interessenlagen, da mit den genannten Technologielösungen neue Unternehmen auftreten, währenddessen das Bewusstsein um eine umweltverträgliche Mobilitätswende steigt und zunehmend auch der Rückbau von Straßenräumen zum Zweck der sanften Mobilität gefordert wird (siehe Abb. 1). 
Neben den genannten Risiken in der Verkehrsinteraktion kann sich eine flächendeckende Ausdehnung von Zu- und Ausstiegssituationen im öffentlichen Raum negativ auswirken. Durch das Halten in zweiter Reihe und die räumliche Ausbreitung des Verkehrsaufkommens sinken Durchlässigkeit und Zugänglichkeit. Im Hinblick auf das automatisierte Fahren ist davon auszugehen, dass dieser Effekt verschärft wird. Hohe Durchdringungsraten von automatisierten Fahrzeugen können aufgrund von Zu- und Ausstiegen Mehrfahrten und Stauaufkommen verursachen, wodurch sich die Querungsmöglichkeiten von bestimmten Straßenräumen signifikant verschlechtern könnten. Dadurch wären schwächere Mobilitätsteilnehmerlnnen klar benachteiligt und öffentliche Räume könnten an Aufenthaltsqualität einbüßen. Zudem kann eine flächendeckende Verteilung von Haltemöglichkeiten zur Konsequenz haben, dass aufgrund des Komfortvorteils die Nachfrage von automatisierten Fahrzeugen auf kurzen Strecken ansteigt und das Verkehrsaufkommen weiter zunimmt (González-González et al. 2020, Kondor et al. 2020, Cavoli et al. 2017).

Eine flächendeckende Ausbreitung von Zu- und Ausstiegssituationen ist auch dahingehend kritisch zu betrachten, dass die erhofften räumlichen Vorteile des automatisierten Fahrens nur realisierbar sein werden, wenn ein Großteil der Fahrzeugkilometer geteilt zurückgelegt wird. In diesem Zusammenhang konnte gezeigt werden, dass die Bündelung von Fahrten und höhere Besetzungsraten durch die Anfahrt von dezidierten Sammelpunkten erleichtert wird, während die Anzahl der gefahrenen Kilometer sinkt (Stiglic et al. 2015).

Vor diesem Hintergrund wird deutlich, dass die Stadtplanung unter den Vorzeichen des automatisierten Fahrens vor der Herausforderung steht, zusehends diverse Nutzungsformen und teils konträre Interessenlagen im öffentlichen Raum in Einklang zu bringen. Dabei ist der steigende Bedarf an temporären Haltemöglichkeiten ebenso zu berücksichtigen wie die Ansprüche der Verkehrssicherheit, der Verkehrsflusseffizienz, der Klimaverträglichkeit und der Stadtraumqualität.

Abbildung 1: Nutzungswandel im öffentlichen Raum
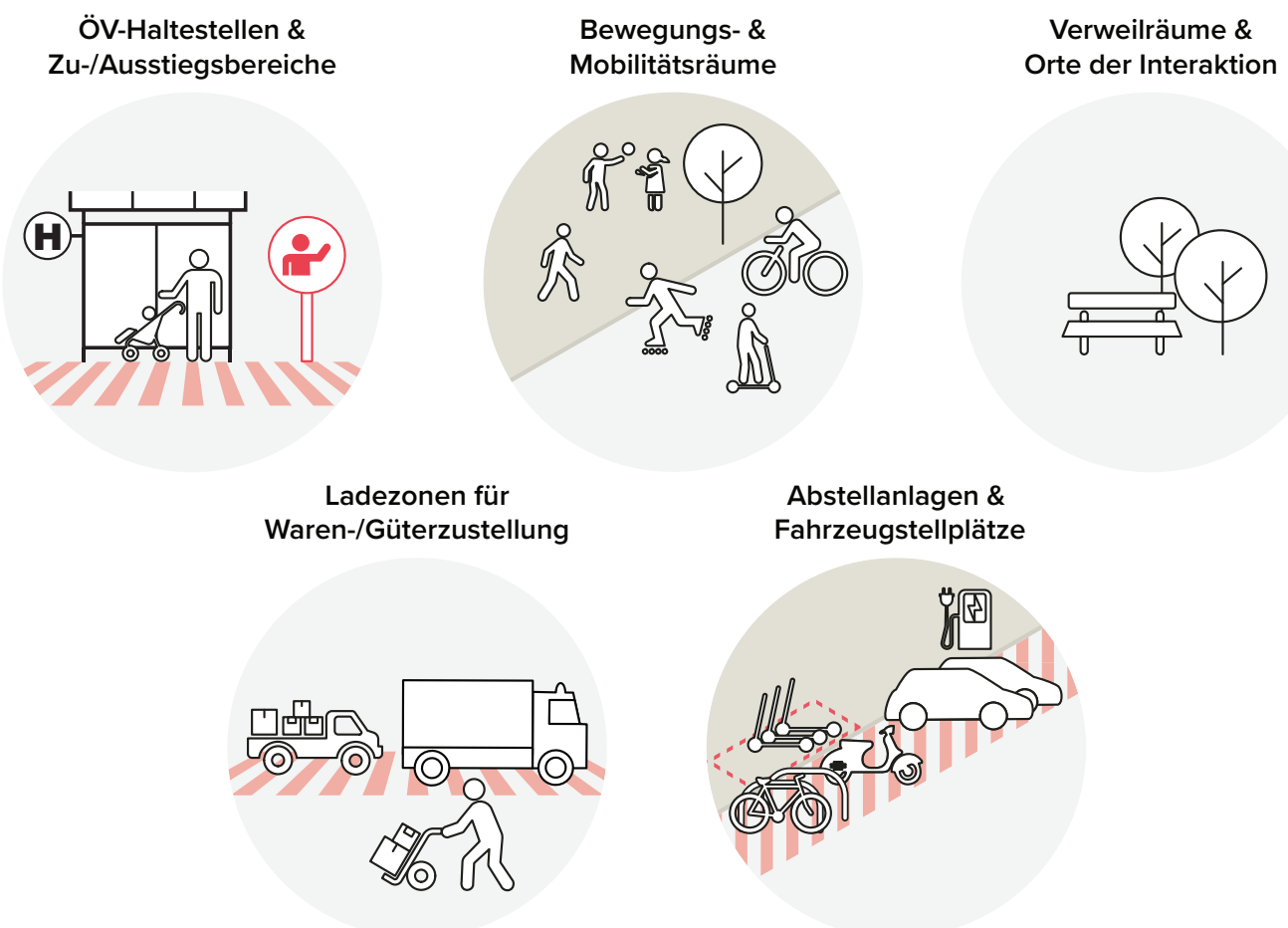

Quelle: eigene Darstellung 


\subsection{BETRACHTUNGSFOKUS}

Ausgehend von der Problematik einer steigenden Flächenkonkurrenz im öffentlichen Raum und von dem Bedarf an integrierten Planungsansätzen wird in den nachstehenden Betrachtungen auf folgende Fragestellungen eingegangen:

- Wo entstehen räumliche und modale Schnittstellen? Worauf ist bei der Planung von Einsatzgebieten und Stationsnetzwerken zu achten?

- Welcher Spielregeln bedarf es, um Schnittstellen im öffentlichen Raum sichtbar zu machen und im Sinne heterogener Mobilitätsformen und Nutzungsansprüche zu gestalten?

- Inwieweit können Schnittstellen der Mobilität positive Impulse für die stadträumliche Entwicklung liefern und als Scharniere im Stadtgefüge nutzbar gemacht werden?

Zudem sind Auswirkungen sowie Handlungsansätze je nach Siedlungsstruktur, Stadtraum und angrenzenden Nutzungen differenziert zu betrachten, denn die Potentiale und Grenzen des automatisierten Fahrens unterscheiden sich zwischen innerstädtischen, suburbanen und ländlichen Gebieten. Dabei sind nicht nur bestehende Faktoren wie die Bebauungsstruktur, der Querschnitt des Straßenraums oder die Nutzungsintensität umliegender Erdgeschosszonen zu berücksichtigen, sondern auch jene stadträumlichen Potentiale, die erst durch die Umverteilung mobilitätsbezogener Flächen wirksam gemacht werden können, wie beispielsweise die Aufwertung der Wahrnehmbarkeit und Aufenthaltsqualität öffentlicher Räume. In den Betrachtungen wird dahingehend zwischen städtischen, suburbanen und ländlichen Räumen differenziert.

\section{STRATEGISCHE RAHMENBEDINGUNGEN RÄUMLICHER SCHNITTSTELLEN}

Eine raumbezogene Chance automatisierter und vernetzter Mobilitätskonzepte beruht auf der Bündelung von Fahrten (Greenblatt/Shaheen 2015, Zhang et al. 2015), sei es in einer Fahrgemeinschaft zwischen Kolleglnnen, in einem Pooling-Service oder der Integration von Personen- und Warenverkehr. Allerdings erfordert die Realisierung damit verbundener räumlicher Vorteile (z. B. der Rückgang des Parkraumbedarfs, das Freiwerden von Bestandsflächen, die Transformation bestehender Strukturen etc.) die Festlegung von zielgerichteten Steuerungsweisen. Üblicherweise werden im Zusammenhang mit dem automatisierten Fahren in etwa Maßnahmen wie der Vorrang für Fahrzeuge mit hohen Besetzungsraten, die streckengebundene Bepreisung oder eine restriktivere Parkraumbewirtschaftung als Mittel genannt, um dem individuellen Nutzen von Fahrzeugen entgegenzuwirken (Sousa et al. 2018, Zhang 2017). Allerdings kann auch mittels räumlicher Strategien die Bündelung von Verkehrsströmen gefördert werden; so beispielsweise durch die Bereitstellung entsprechender Mobilitätsinfrastruktur oder durch die Konzentration von Angeboten und Aktivitäten, wodurch Wege räumlich und zeitlich zusammengeführt werden. In beiderlei Hinsicht entstehen räumliche Schnittstellen, an denen unterschiedliche Anforderungen in Einklang zu bringen sind und Bestand mit Zukünftigem zu integrieren ist.

An welcher Stelle neue räumliche Schnittstellen entstehen und in welchem Ausmaß sie Siedlungs- und Stadtraumstrukturen prägen werden, wird auf einer übergeordneten Ebene durch die strategischen Leitlinien und rahmengebenden Steuerungsweisen des automatisierten Fahrens 
beeinflusst. Dies betrifft allem voran die Einsatzgebiete automatisierter Flotten, wobei besonders die Eignung der AngebotsformenOF1 einer räumlich differenzierten Betrachtung bedarf. Weiters stellt die Netzintegration automatisierter Angebotsformen eine Voraussetzung für die Standortplanung von Halte- und Umstiegspunkten dar. Wie bereits erwähnt, konnten Modellierungsstudien zwar zeigen, dass die Einrichtung von Sammelpunkten die Fahrtenbündelung automatisierter Mobilitätsdienste erleichtern kann (Stiglic et al. 2015), doch mangelt es international betrachtet, neben einzelnen Pilotvorhaben, an regulatorischen Ansätzen für die strategische Planung von dezidierten Haltezonen und Sammelpunkten (ITF 2018: 58; Schaller 2019). Nicht zuletzt sind auf strategischer Ebene auch Fragen der Zentrenentwicklung im Umfeld neuer Knotenpunkte und der langfristigen Anpassungsfähigkeit von Infrastruktur und Stadträumen zu berücksichtigen. Die genannten Aspekte werden in Folge vertiefend erläutert.

\subsection{BEWERTUNGEN INTEGRIEREN: EINSATZGEBIETE, MODI UND ZULÄSSIGE GESCHWINDIGKEITEN}

In Anbetracht einer möglicherweise langfristigen Übergangsphase, in deren Rahmen automatisierte Fahrzeuge lediglich in dafür angemessenen Bereichen zum Einsatz kommen werden, ist eine raumbezogene Bewertung und Priorisierung von Einsatzgebie-

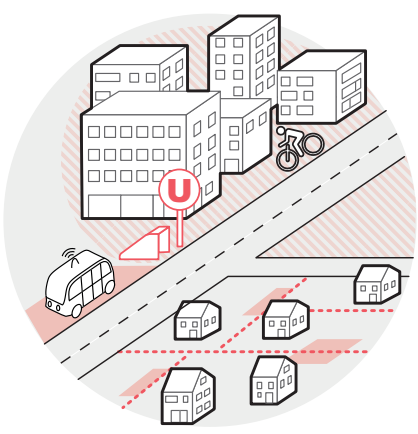
ten, Modi und zulässigen Geschwindigkeiten erforderlich (siehe auch Beitrag 5 von Soteropoulos in diesem Band). Hierzu sind neben klassischen Einsatzkriterien unterschiedlicher Verkehrsmittel auch räumliche Wirkungsweisen der digitalen Vernetzung und Automatisierung zu berücksichtigen, die in vorhandenen Studien in Ansätzen für innerstädtische Gebiete behandelt werden, wobei nicht systematisch zwischen Stadtraumtypen oder auch StraBentypologien differenziert wird. González-González et al. (2020: 9) zeichnen beispielsweise ein Zukunftsbild, wonach sogenannte "Core Attractive Mixed-use Spaces (CAMS)" in städtischen Kerngebieten ausgewiesen werden. Innerhalb dieser Zonen würde automatisiertes Fahren nur eingeschränkt zugelassen und stattdessen öffentlicher Raum rückgewonnen werden. Die Festlegung von räumlich abgegrenzten Zonen dieser Art hat einen grundlegenden Einfluss auf die Lage von räumlichen Schnittstellen. Sowohl innerhalb des Einsatzgebietes als auch an dessen Rändern entsteht der Bedarf nach Umstiegspunkten und Übergangszonen, an denen unterschiedliche Modi räumlich, zeitlich und tariflich aufeinander abgestimmt werden.

Um mögliche Einsatzgebiete unter Berücksichtigung stadträumlicher Effekte auszuweisen, bedarf es eines Verständnisses der raumstrukturellen Eignung neuer Mobilitätskonzepte. In der Verkehrsplanung werden für die Festlegung des Verkehrsmitteleinsatzes klassischerweise folgende Kriterien berücksichtigt: Wirtschaftlichkeit, Funktionalität (Beförderungsgeschwindigkeit und Kapazität), Angebotshierarchie sowie siedlungsräumliche Zweckmäßigkeit (Weidmann et al. 2011). Um Letztere zu ermitteln, werden die Einwohner- und die Nutzungsdichte als wichtigste Parameter neben den Siedlungsgrößen und Kernnutzungsdichten genannt. Nach Weidmann et al. (2011) ist anhand dieser Dimensionen abzuleiten, welche räumlichen Strukturtypen den

1 Bereits heute vervielfältigen sich Angebotsformen des ÖPNV und der privaten Dienstleister. Grob können diese zwischen liniengebundenen und flexiblen Angebotsformen unterschieden werden. Unter Berücksichtigung der Automatisierung des Mobilitätswesens kann von folgenden Angebotsformen ausgegangen werden: AV-Gelenkbus, AV-Standardbus, AV-Minibus/Shuttle, AV-Van (Ride-Sharing), AV-Sammeltaxi (Ride-Sharing), AV-Einzeltaxi (Car-Sharing; Soteropoulos et al. 2019, siehe Beitrag 18 von Stickler in diesem Band). 
Einsatz bestimmter Verkehrssysteme begünstigen. Beispielsweise setzen hochleistungsfähige Verkehrssysteme (z. B. U-Bahn oder S-Bahn) Siedlungsräume hoher Einwohnerdichte und ausdifferenzierte Korridore voraus. Indessen rechtfertigen disperse Räume aufgrund geringer Nachfrage oder besonderer topographischer Bedingungen meist keine schienengebundenen Systeme und erfordern stattdessen kleinere Fahrzeugeinheiten für Erschließungs- und Verteilersysteme sowie Zubringerdienste zu höherrangigen Knotenpunkten (Weidmann et al. 2011). Insbesondere hierzu werden automatisierte Angebotsformen als Lösung angesehen, da von einer höheren Wirtschaftlichkeit und von flexiblen Bedienformen ausgegangen wird. Darüber hinaus wird im Kontext der integrierten Stadt- und Mobilitätsentwicklung insbesondere der Grad der funktionalen Durchmischung berücksichtigt. In diesem Zusammenhang können beispielsweise Entwicklungspotentiale innerhalb von Einzugsgebieten des öffentlichen Nahverkehrs ausgelotet oder das Verhältnis von Stationsumfeld und Verkehrsnachfrage untersucht werden (Bertolini 1999). Der Einsatz automatisierter Angebotsformen und die Planung von Schnittstellen ist demnach im Verhältnis zu strukturellen Faktoren wie der Einwohner- und Arbeitsplatzdichte sowie der Diversität von Umfeldnutzungen, aber auch zu Faktoren wie der Verfügbarkeit des ÖPNV und alternativer Modi zu bewerten.

Vor dem Hintergrund der genannten Eignungskriterien sind zwei wesentliche Veränderungstendenzen im Zusammenhang mit dem automatisierten Fahren zu berücksichtigen, durch die eine Bewertung von dessen Zweckmäßigkeit an Komplexität gewinnt: Zunächst gilt es festzuhalten, dass sich aufgrund neuer Akteure am Mobilitätsmarkt die Grenze zwischen öffentlichen und privaten Angeboten zunehmend auflöst (Lenz/Fraedrich 2015; Mitteregger et al. 2020: 44). Eine neu auftretende Konkurrenzsituation zwischen öffentlichem Linienbetrieb und flexiblen Bedarfsdiensten privater Fahrdienstleister hat zur Folge, dass in bestimmten Gebieten die für den öffentlichen Verkehrsbetrieb notwendigen Nachfragedichten sinken könnten. Um das öffentliche Verkehrsnetz als Rückgrat der Mobilität zu erhalten, sind Einsatzgebiete automatisierter Mobilitätsdienste auf Basis des bestehenden ÖV-Netzes zu bestimmen und jene Siedlungsräume zu priorisieren, in denen bestehende Lücken geschlossen bzw. Schwachstellen bedarfsorientiert gestärkt werden können. Speziell für Räume mittlerer bis geringer Dichte kann dies bedeuten, dass die Einzugsgebiete von überörtlichen Verkehrsknoten um die Reichweite von automatisierten Zubringerdiensten erweitert und neu ausgewiesene Routen als Transformations- und Entwicklungsräume priorisiert werden. Auch impliziert dies die Notwendigkeit, neue Schnittstellen als Übergänge und Knoten räumlich zu integrieren, sodass die Inanspruchnahme von intermodalen Wegen für Nutzerlnnen erleichtert wird.

Zudem ist die Zweckmäßigkeit von automatisierten Angebotsformen angesichts möglicher Wirkungen im öffentlichen Raum zu beurteilen, die je nach Gebietstyp und Straßenraum variieren können. Hierzu dient beispielsweise die Überprüfung der straßenräumlichen Verträglichkeit auf Ebene des Quartiers oder Ortsteils (siehe Beitrag 5 von Soteropoulos in diesem Band). Dabei ist zu untersuchen, inwieweit die Zulassung einzelner Modi und Geschwindigkeiten zu Trennwirkungen, im Sinne erschwerter Querungsmöglichkeiten für den Fuß- und Radverkehr, in bestimmten Straßentypologien (z. B. Wohnstraßen, Quartiersstraßen, Einfallstraßen/Hauptverkehrsstraßen, Verbindungsstraßen etc.) führen kann. Darüber hinaus ist die Flächeninanspruchnahme, die durch den Anstieg von kurzfristig haltenden und parkenden Fahrzeugflotten entstehen kann, zu ermitteln. Beide Wirkungsformen sind im Verhältnis zu den bestehenden Nutzungsansprüchen angrenzender Funktionen zu bewerten. So schafft beispielsweise eine innerstädtische Quartiersstraße mit einer Konzentration von Einzelhandel und Gastronomie andere Nutzungsdichten und räumliche Anforderungen (z. B. Querungsmöglichkeiten, Schanigärten, Verweilräume etc.) als eine reine Wohnstraße oder Hauptverkehrsachse am Stadtrand. Auf dieser Basis wird zu prüfen sein, ob bestimmte Anwendungsformen, wie beispielsweise der automatisierte Lieferverkehr, aufgrund von Nutzungskonflikten von gewissen Straßenkategorien gänzlich auszunehmen oder nur zeitlich einzuschränken ist (siehe Beitrag 7 von Leerkamp et al. in diesem Band). Auch wäre in diesem Sinne abzuwägen, inwieweit es dienlich sein kann, 
plattformbasierte Mitfahrdienste auf ausgewiesenen Bus- und Taxispuren zuzulassen. Dies kann im Zuge der Übergangsphase zwar einen Anreiz darstellen, Mobilitätsdienste anstatt Privatfahrzeuge zu nutzen, je nach Straßenraum kann dies allerdings auch zu einer Behinderung des ÖPNV und der Querungsmöglichkeiten von Fuß- und Radfahrenden beitragen.

Tabelle 1: Bewertungen integrieren: Einsatzgebiete, Modi und zulässige Geschwindigkeiten

\section{Räumlich differenzierte Betrachtung}

\begin{tabular}{ll}
\hline Städtischer Raum & $\begin{array}{l}\text { In Räumen hoher Dichte wird es bedeutsam sein, das bestehende ÖPNV-Angebot zu } \\
\text { priorisieren und es lediglich in ausgewählten Randgebieten und Randzeiten durch } \\
\text { automatisierte Bedarfsbusse zu ergänzen. }\end{array}$ \\
\hline Suburbaner Raum & $\begin{array}{l}\text { In suburbanen Achsenzwischenräumen könnten selbstfahrende und flexibel } \\
\text { eingesetzte Angebotsformen dazu dienen, die Feinerschließung in der Fläche zu } \\
\text { decken und eine Alternative zum Automobil zu schaffen (Mitteregger et al. 2020). Die } \\
\text { Möglichkeiten, Wegstrecken räumlich und zeitlich zu bündeln, fallen höher aus als in } \\
\text { ländlichen Räumen. }\end{array}$ \\
\hline Ländlicher Raum & $\begin{array}{l}\text { Da sich die Verkehrswege in Siedlungsräumen geringer Dichte räumlich und zeitlich } \\
\text { divers gestalten, stellen flexible Bedienformen automatisierter Shuttle eine Chance } \\
\text { dar, bestehende Lücken im ÖPNV-Netz zu schließen und die öffentliche Anbindung zu } \\
\text { sichern (Jürgens 2020). }\end{array}$ \\
\hline
\end{tabular}

\subsection{STATIONSNETZE ERWEITERN: AUSBAU UM HALTEZONEN UND SAMMELPUNKTE}

Angesichts steigender Mobilitätsoptionen sind Städte angehalten, unterschiedliche Angebotsformen aufeinander abzustimmen und dezidierte Sammelpunkte als Teil einer strategischen Netzplanung zu berücksichtigen. Hierbei ist zwischen einer klassischen Netzbildung für den ÖPNV, wonach einzelne Linien zu einem meist hierarchischen Streckennetz integriert werden, und

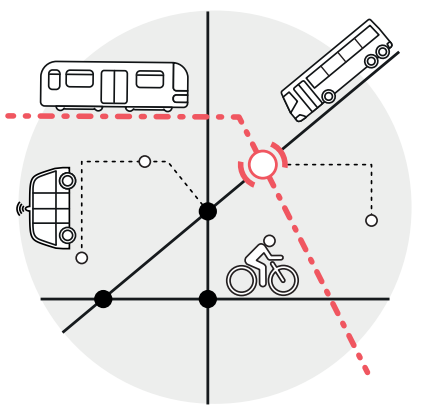
einer homogenen Netzabdeckung flexibler (bedarfsorientierter) Bedienformen zu differenzieren (Schnieder 2018). Letztere zeichnet sich durch die Zielsetzung aus, Direktverbindungen zwischen Quell- und Zielorten anzubieten, und erfordert dementsprechend eine kleinteilige Verortung von Sammelpunkten in der Fläche. In einer Modellierungsstudie zu den straßenräumlichen Auswirkungen von flexiblen Sharing-Angeboten wurde ein möglicher Ansatz hierzu erforscht (ITF 2018). Für ein innenstädtisches Gebiet Lissabons, ein wirtschaftliches und gewerbliches Zentrum, wurde ermittelt, dass die Bedarfsabdeckung eine gleichmäßige Verteilung von Haltezonen an Quell- und Zielorten im gesamten Straßennetz erfordern würde. Als Indikator für den verkehrlichen Bedarf wurde das tageszeitliche Stauaufkommen sowie dessen Dauer angenommen (ITF 2018: 70). Im Modell wurden demnach am Anfang und Ende jedes Häuserblocks, zur Mitte von besonders langen Straßenzügen und an Orten hoher Verkehrsnachfrage (z. B. Einkaufszentren, Schulen etc.) Haltezonen als Zu- und Ausstiegsbereiche verortet, um eine möglichst hohe Netzabdeckung zu erzielen (Abb. 2).

\section{Integration in bestehende Stationsnetze}

Ähnlich den Einsatzgebieten ist auch die Dichte von Sammelpunkten kontextspezifisch zu planen. Dabei sind die jeweiligen Siedlungsstrukturen und Straßentypologien sowie Nutzungsdichten und Verfügbarkeiten des öffentlichen Verkehrsnetzes zu berücksichtigen. In dichten 
Siedlungsgebieten mit einem ausgebauten öffentlichen Verkehrsnetz ist neben der Orientierung an Quell- und Zielorten sowie Orten hoher Nachfrage (z. B. Flughäfen, Geschäftsviertel, Einkaufszentren etc.) die Integration in die bestehende Netzhierarchie des ÖPNV ausschlaggebend. Der Haltestellenabstand bestimmt dabei nicht nur die hierarchische Funktionszuweisung im Gesamtverkehrssystem, sondern auch potentielle Nutzungsraten. So erzeugen dichte Stationsnetze, wie beispielsweise das für Lissabon angenommene Netz mit einem Abstand von 100 bis 200 Metern, eine tendenziell höhere Nachfrage. Im Sinne einer Priorisierung umweltverträglicher Mobilitätsformen kann ein Netzkonzept mit vergleichbar geringen Haltestellenentfernungen durchaus kritisch betrachtet werden. In innerstädtischen Lagen könnte dies zu einer Schwächung des ÖPNV und insbesondere der aktiven Mobilität führen, währenddessen es sich in Stadtrandlagen sowie in Gebieten geringer ÖPNV-Abdeckung empfiehlt, Möglichkeiten auszuloten, durch dicht geplante Sammelpunkte Netzlücken zu schließen.

Abbildung 2: Standorte von Zu- und Ausstiegspunkten für geteilte Mobilitätsdienste in Lissabon

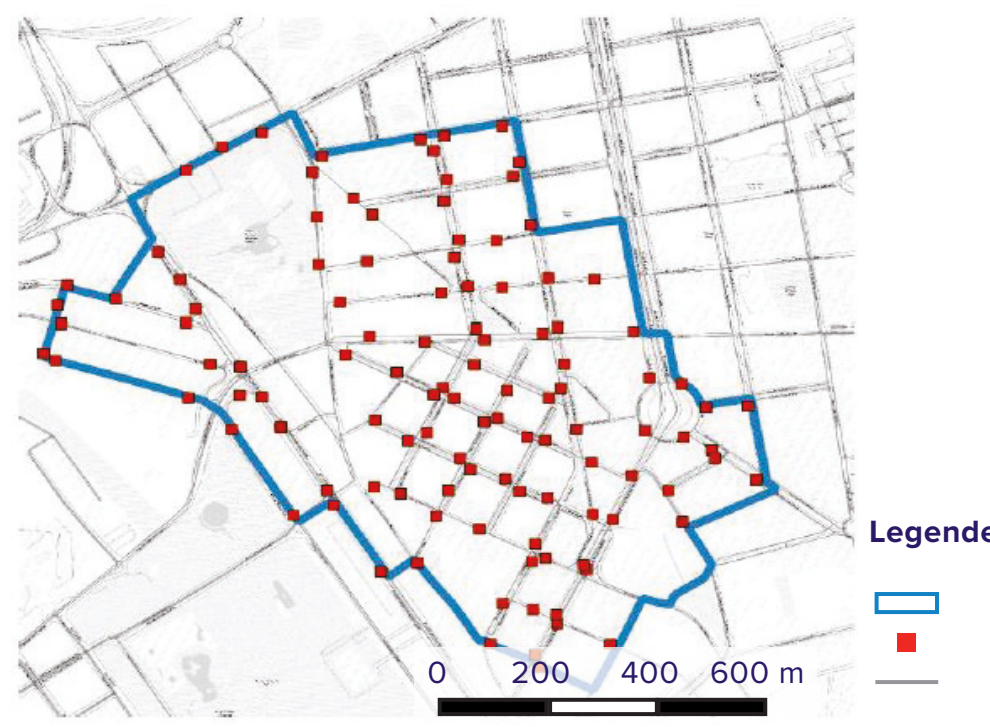

Untersuchungsgebiet Pick-up und drop-off Areale Straßennetz

Quelle: ITF (2018: 67)

Dementsprechend sind innerstädtisch in erster Linie vorhandene Stationsnetze um Haltezonen für neue Mobilitätsdienste zu erweitern. Diese bestehen in der Regel aus höherrangigen Stationen (Bahnhöfe und U-/S-Bahn-Stationen), mittleren und kleineren Knoten (Straßenbahnstationen und Linienkreuzungen) sowie einzelnen Haltestellen. An diesen Orten entstehen unterschiedliche Schnittstellen, die städtebaulich wie siedlungsstrukturell zu integrieren sind und gestalterisch ganz grundlegend verschieden sein können (Abb. 3). Im Gegensatz zur Entwicklung des Schienenverkehrs im 19. Jahrhundert und der Errichtung von Bahnhöfen entsteht dadurch weniger ein neuer „Typus“ an Infrastrukturgebäuden als vielmehr die Notwendigkeit, die Vielfalt unterschiedlicher Mobilitätsangebote räumlich wie digital miteinander zu verknüpfen. Dennoch ist nicht auszuschließen, dass Orte wie Parkgaragen, die bisher eine geringe Aufmerksamkeit erhielten, durch Nutzungsanreicherungen zu neuen Knotenpunkten transformiert werden.

Die Flächenerfordernisse für Haltezonen und Sammelpunkte automatisierter Angebotsformen können je nach Nutzungsform und Nachfrage drastisch variieren. So ergab etwa eine Studie für Schweizer Regionalbahnhöfe, dass die erforderlichen Haltezonen für automatisierte Bussysteme flächenmäßig wesentlich geringer ausfallen, als wenn sich automatisierte Privatfahrzeuge oder Car-Sharing als Zubringer durchsetzen würden (Sinner et al. 2018). Dies ist einerseits auf die geringere Anzahl an Fahrzeugen zurückzuführen, die ein automatisiertes Bussystem er- 
Abbildung 3: Integration von räumlichen Schnittstellen an unterschiedlichen Stationstypen, die nach Verkehrsraumtypen kategorisiert sind

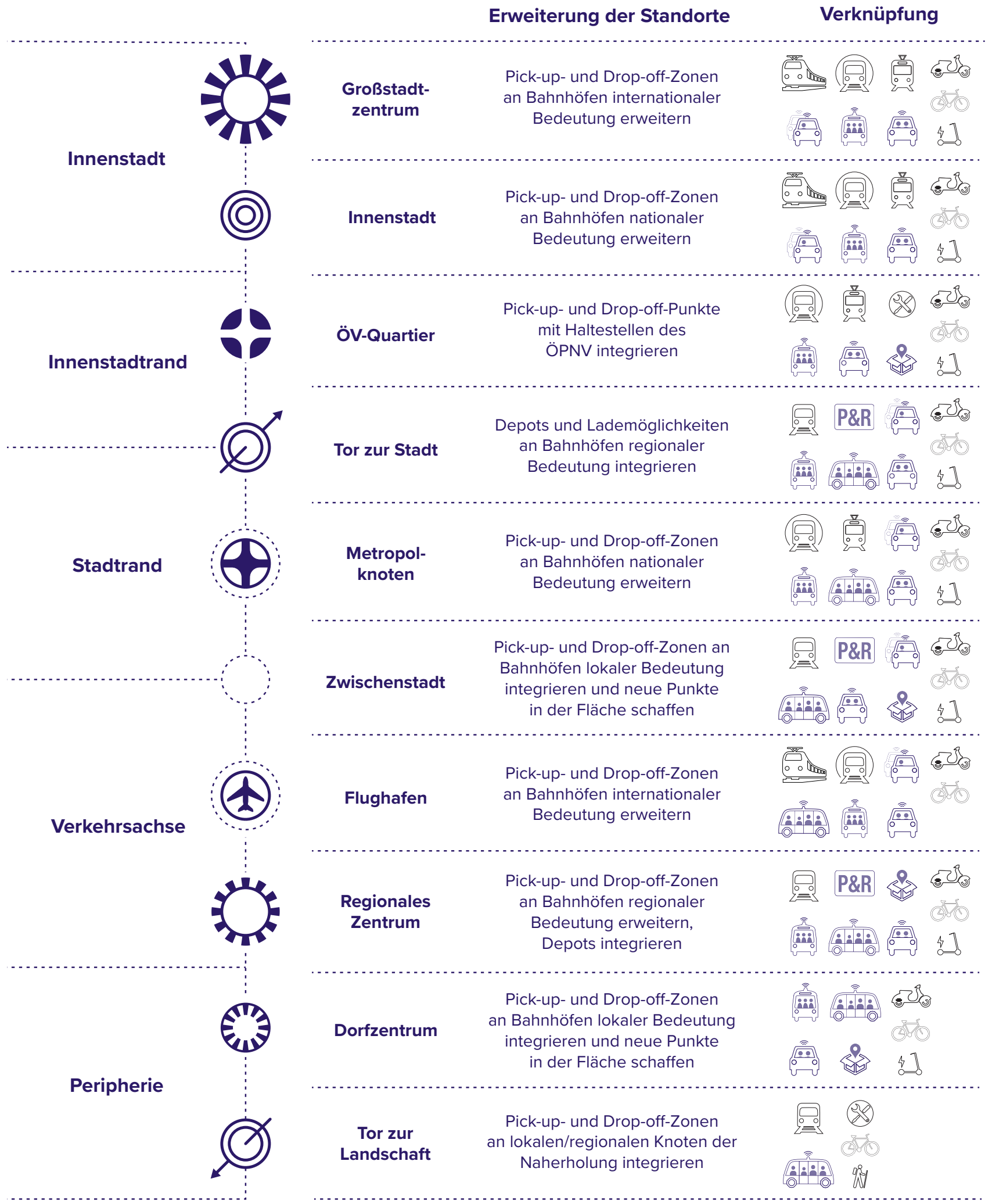

Quelle: eigene Darstellung nach Ram et al. (2013: 100) und Matthes/Gertz (2014: 61) 
fordern würde, und andererseits auf die Möglichkeit, dass aufgrund von planmäßigen An- und Abfahrtszeiten Haltezonen dem tatsächlichen Bedarf entsprechend dimensioniert werden können (ebd.). Indessen zeichnet das Ausmaß an Halte- und Parkflächen, das für ein auf automatisiertem Car-Sharing basiertes System ermittelt wurde, ein kaum nachhaltiges Zukunftsbild. Die enormen Flächenerfordernisse würden nicht zuletzt die räumlichen Möglichkeiten einschränken, höherrangige Knotenpunkte als fußläufig erreichbare Zentren aufzuwerten.

\section{Netzerweiterung durch automatisierte Angebotsformen}

In suburbanen und ländlich-peripheren Gebieten, die aufgrund einer geringen Netzabdeckung häufig schwer erreichbar sind, ermöglichen automatisierte Angebotsformen die Erweiterung bestehender Stationsnetze. Dabei gilt es, bei der Routen- und Netzplanung bestehende Zentrenstrukturen besonders zu berücksichtigen. Anstatt alte Ortskerne zugunsten der Effizienz zu umfahren, kann ein ausgewogenes Verhältnis zwischen einer Bedienung und der Vermeidung von verkehrlichen Trennwirkungen zu einer Stabilisierung und Aufwertung bestehender Strukturen beitragen (vgl. Angélil et al. 2012). Aufgrund der vielerorts zeitlich stark schwankenden Mobilitätsnachfrage ist allerdings zu prüfen, inwiefern bestimmte Streckenabschnitte tageszeitabhängig zu bedienen und Anfahrtspunkte dynamisch bereitzustellen sind. Beispielsweise können dezidierte Sammelpunkte vor allem untertags angefahren werden, um die Fahrtenbündelung zu erleichtern, während zu Tagesrandzeiten ein komfortabler Tür-zu-Tür-Betrieb angeboten wird. Überall dort, wo das öffentliche Verkehrsnetz durch automatisiert betriebene, bedarfsgerechte Shuttlebusse oder ausgedehnte Betriebszeiten erweitert wird, ist auch das sichere Zu- und Aussteigen sowie das komfortable Umsteigen auf ein Fahrrad oder Pedelec zu gewährleisten. Ein integriertes Mobilitätsmanagement und Kooperationen der öffentlichen Hand mit privaten Mobilitätsdienstleistern sind wesentliche Voraussetzungen hierfür.

Tabelle 2: Stationsnetze erweitern: Ausbau um Haltezonen und Sammelpunkte

\section{Räumlich differenzierte Betrachtung}

\begin{tabular}{ll}
\hline Städtischer Raum & $\begin{array}{l}\text { In dichten Siedlungsgebieten sind Haltezonen für automatisierte Angebotsformen in } \\
\text { das bestehende Stationsnetz des ÖPNV zu integrieren und neue Sammelpunkte nur } \\
\text { im Bedarfsfall zu ergänzen. }\end{array}$ \\
\hline Suburbaner Raum & $\begin{array}{l}\text { Die oftmals unzureichende Erschließung suburbaner Gebiete erfordert eine } \\
\text { attraktive Nachverdichtung des Stationsnetzes in der Fläche. Mit automatisierten } \\
\text { Nachbarschaftsbussen können Achsenzwischenäume tangential und auf einem } \\
\text { feinmaschigen Stationsnetz basierend bedient werden. }\end{array}$ \\
\hline Ländlicher Raum & $\begin{array}{l}\text { In ländlich-peripheren Räumen sind besonders entlegene Siedlungsräume durch } \\
\text { bedarfsorientierte Angebotsformen zu erschließen. Für eine attraktive Gestaltung von } \\
\text { automatisierten Angebotsformen sind Halte- und Sammelpunkte wohnortnah zu planen. }\end{array}$ \\
\hline
\end{tabular}

\subsection{ZENTRALITÄTEN STÄRKEN: VERKNÜPFUNG VON NUTZUNGEN, WEGEN UND ZIELEN}

Im Sinne einer „Stadt und Region der kurzen Wege“ (Beckmann et al. 2011: 63) sind auch Stationsnetze automatisierter Angebotsformen mit der Entwicklung durchmischter Zentren integriert zu planen. Insbesondere nachdem das automatisierte Fahren das Risiko birgt, aufgrund genannter Komfortvorteile den Flächenverbrauch durch Zersiedlungstendenzen zu begünstigen. Risiken des indu-

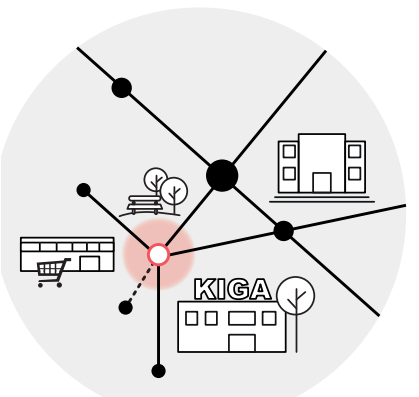


zierten Verkehrs (z. B. aufgrund von Mobilitätsverlagerungen oder neu akquirierter Nutzergruppen) gilt es durch Strategien der Verkehrsvermeidung gegenzusteuern (Hörl et al. 2019). Ähnlich dem Ausbau von S-Bahn-Strecken, der in der Vergangenheit oftmals für die Innenentwicklung stationsnaher Gebiete impulsgebend war (ARE 2004, Weidmann et al. 2011), ist auch in Anbetracht automatisierter Mobilitätsnetze zu berücksichtigen, inwiefern neu erschlossene Strukturen funktional angereichert und als Zentren entwickelt werden können. Die funktionale Verdichtung im Umkreis von Verkehrsknoten kann dabei zu einer Aufwertung des Standortes beitragen und einen Anreiz für den Zugriff auf Sharing-Angebote liefern. Auf der Maßstabsebene des Quartiers oder Ortsteils ist zu berücksichtigen, inwiefern auch eine quartierstrennende Wirkung von Infrastrukturen abgeschwächt und neue räumliche Verbindungen hergestellt werden können. Besonders in suburbanen und ländlich-peripheren Gebieten, jenen Räumen, in denen die größten Erreichbarkeitsgewinne durch die automatisierte Mobilität erwartet werden, ist anzudenken, dass durch die Planung von Mobilitätsknoten an den Schnittstellen von Siedlungsräumen ein Impuls für die Siedlungsentwicklung gesetzt werden kann (vgl. Kretz/Kueng 2016, Bruck 2019).

Die Entwicklung von Zentralitäten gewinnt auch im Hinblick auf ein attraktives Umsteigen und die Akzeptanz intermodaler Wege an Bedeutung. Hierzu ist neben kurzen Fußwegen besonders die Anlagerung von Alltagsnutzungen im Umkreis von Mobilitätsknoten zu berücksichtigen. Lassen sich Wegzwecke, beispielsweise die Besorgung von Lebensmitteln und der Arbeitsweg, unkompliziert miteinander kombinieren, entstehen einerseits Zeitersparnisse, andererseits wird die Bedeutung des Ortes in den alltäglichen Netzwerken von Personen begünstigt (Kretz/Kueng 2016: 46).

Durch die Integration einer Vielfalt unterschiedlicher Nutzungen kann ein Mobilitätsknotenpunkt zu unterschiedlichen Tageszeiten belebt und erlebbar gemacht werden (vgl. ebd.). Möglichkeiten für Synergien entstehen zudem aufgrund des fortschreitenden Trends, mittels digitaler Vernetzung städtische Funktionsräume und Prozesse dezentral auszulagern. So gewinnt beispielsweise die Einrichtung von Co-Working-Spaces sowie hybriden Büro- und Dienstleistungsangeboten im Kontext einer polyzentrischen Dezentralisierung und zunehmend flexibler Lebensstile an Bedeutung, die vor dem Hintergrund der automatisierten Mobilität auch als Teil einer integrierten Planung von neuen Stationsnetzen, lebendigen Zentren und starken Siedlungsrändern zu berücksichtigen sind.

Tabelle 3: Zentralitäten stärken: Verknüpfung von Nutzungen, Wegen und Zielen

\section{Räumlich differenzierte Betrachtung}

\begin{tabular}{ll}
\hline Städtischer Raum & $\begin{array}{l}\text { In der Regel weisen Räume hoher Siedlungsdichte bereits eine Vielzahl an } \\
\text { Zentralitäten auf. So kann es innerstädtisch vielmehr notwendig sein, durch die } \\
\text { Verlagerung von Sammelpunkten bestehende Zentren zu entlasten oder effektiver } \\
\text { miteinander zu verknüpfen. }\end{array}$ \\
\hline Suburbaner Raum & $\begin{array}{l}\text { In suburbanen Räumen können durch das Freiwerden von Parkplatzflächen (siehe } \\
\text { Beitrag 15 von Mitteregger und Soteropoulos in diesem Band) Möglichkeitsräume } \\
\text { entstehen, um die an Mobilitätsknoten angrenzenden Gebiete funktional zu } \\
\text { durchmischen, nachzuverdichten und durch Freiräume aufzuwerten. }\end{array}$ \\
\hline Ländlicher Raum & $\begin{array}{l}\text { Neben der Herausforderung trennender Distanzen sind ländliche Räume oftmals von } \\
\text { einem Trend der Abwanderungs- und des Leerstands geprägt. Auch hier kann die } \\
\text { gezielte Aufwertung von Mobilitätsknoten einen wichtigen Impuls für kleinräumige } \\
\text { Zentren liefern. }\end{array}$ \\
\hline
\end{tabular}




\subsection{ADAPTIVITÄT SICHERN: OFFENHEIT FÜR ZUKÜNFTIGEN NUTZUNGSWANDEL}

Angesichts der Unbestimmbarkeit der Entwicklungsrichtung sowie der Folgen des automatisierten Fahrens drängt sich das planerische Prinzip der Adaptivität auf. Gemeint ist eine unverbaute Offenheit für Kommendes ebenso wie für die Veränderbarkeit von urbanen Räumen und deren Nutzungsweisen (Taut 1977, Kretz/Kueng 2016). Da derzeit nicht festzumachen ist, welche Szenarien und

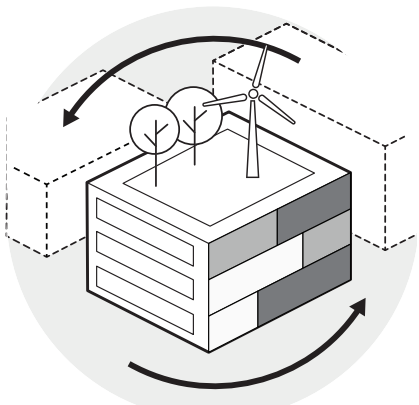
welche Angebotsformen der automatisierten Mobilität sich durchsetzen werden, besteht die Herausforderung, für unterschiedliche Zukünfte zu planen.

Mit Blick auf räumliche Schnittstellen künftiger Mobilitätsnetze bedeutet dies, dass eine hohe Anpassungsfähigkeit oder gar Rückbaubarkeit von Mobilitätsinfrastrukturen, öffentlichen Räumen und baulichen Strukturen zu gewährleisten ist. So ist etwa dafür Sorge zu tragen, dass heutige Park-and-Ride-Anlagen oder Hochgaragen künftig für andere Zwecke umgenutzt werden können. Für den Fall, dass ein weitgehender Mobilitätsumstieg auf Mitfahrdienste und den Umweltverbund einen Rückgang des Parkraumbedarfs zur Folge hätte, könnten darin Ladestationen für elektrische Flotten oder dezentrale Logistikzentren eingerichtet werden (vgl. Lewis/ Anderson 2020: 104; Bienzeisler et al. 2019) - auch da lokale Verteilungszentren eine wichtige Voraussetzung für eine Warenzustellung mittels umweltverträglicher Mobilitätsformen auf der letzten Meile darstellen (González-González et al. 2020). Langfristig könnten aufgrund gänzlich neuer Funktionen allerdings auch Flächenerfordernisse entstehen, die aus heutiger Sicht nicht zu antizipieren sind. Dazu bedarf es Flächenreserven im Stationsumfeld und der Sicherstellung von Spielräumen für künftige Nutzungsanforderungen und Verdichtungsprozesse.

Auf kleinräumiger Ebene des öffentlichen Raums kann eine bauliche und nutzungsbedingte Anpassungsfähigkeit durch eine modulare Gestaltung oder tageszeitabhängige Verordnungen geregelt werden. Indem vermieden wird, öffentliche Räume gestalterisch zu überdeterminieren, kann ein Rahmen für Aneignungen geboten werden und ein Wechselspiel zwischen permanenten und temporären Aktivitäten entstehen. So kann beispielsweise ein Straßenraum zu bestimmten Tageszeiten und bei entsprechendem Fahrverbot als räumliche Erweiterung eines

Tabelle 4: Adaptivität sichern: Offen für zukünftigen Nutzungswandel

\section{Räumlich differenzierte Betrachtung}

\begin{tabular}{ll}
\hline Städtischer Raum & $\begin{array}{l}\text { Die anpassungsfähige und offene Gestaltung öffentlicher Räume spielt vor dem } \\
\text { Hintergrund eines hohen Nutzungsdrucks und einer geringen Flächenverfügbarkeit } \\
\text { in innerstädtischen Gebieten eine besondere Rolle. Der Vielfalt unterschiedlicher } \\
\text { Anforderungen kann nur durch eine möglichst geringe funktionale Festlegung } \\
\text { Rechnung getragen werden. }\end{array}$ \\
\hline Suburbaner Raum & $\begin{array}{l}\text { Im Bewusstsein um den enormen Ressourcenverbrauch suburbaner Gebiete gilt es, der } \\
\text { Anpassungsfähigkeit und Umnutzbarkeit von Strukturen besondere Aufmerksamkeit zu } \\
\text { schenken. Dazu zählt beispielsweise die künftige Transformation von Park-and-Ride- } \\
\text { Anlagen, weitläufigen Parkplatzflächen oder ehemaligen Bahnanlagen. }\end{array}$ \\
\hline Ländlicher Raum & $\begin{array}{l}\text { In ländlichen Räumen besteht das Potential der baulichen, funktionalen und } \\
\text { landschaftlichen Reaktivierung. So können leerstehende Strukturen durch verbesserte } \\
\text { Erreichbarkeiten und Umnutzungen wiederbelebt und als Teil von Kulturlandschaften } \\
\text { erfahrbar gemacht werden. }\end{array}$ \\
\hline
\end{tabular}


Schulhofs dienen oder an bestimmten Wochentagen für Märkte und kulturelle Veranstaltungen nutzbar gemacht werden (Bendiks/Degros 2019). Werden zudem Möglichkeiten der digitalen Vernetzung mit der räumlichen Gestaltung integriert, könnten beispielsweise Haltezonen dynamisch und bedarfsabgestimmt verfügbar gemacht und Fahrzeuge davon digital in Kenntnis gesetzt werden, was eine flexible Routenführung zu Tagesrandzeiten oder eine alternative Flächennutzung zu Schwachlastzeiten erleichtern könnte.

\section{KLEINRÄUMIGE GESTALTUNG MULTIMODALER ÖFFENTLICHER RÄUME}

Veränderungen im öffentlichen Raum erfordern neben strategischen Rahmenbedingungen auch die Festlegung von klaren Gestaltungsansätzen und Zielen. Der begonnene Wandel im Mobilitätswesen schafft einen Anlass, die aktuelle Nutzungsverteilung des öffentlichen Raums im Sinne einer nachhaltigen Mobilität neu zu denken. Anstatt den Stadtraum der technologischen Eignung automatisierter Fahrzeuge anzupassen, wie es beispielsweise hinsichtlich der Bereitstellung von einheitlichen Fahrbahnmarkierungen oder Sensoren diskutiert wird, können Kommunen durch räumliche Anforderungen und Zielsetzungen die Entwicklung der Technologie mitbeeinflussen. In Folge werden Verkehrsanlagen, Freiräume und die umliegende Bebauung als integrale Elemente des öffentlichen Raums verstanden, um sowohl Wirkungsweisen als auch Handlungsansätze für den Einsatz automatisierter Angebotsformen integriert zu betrachten.

Zeitnah betreffen die raumprägendsten Veränderungen vor allem die Organisation des kurzfristigen Haltens und der Umstiegsmöglichkeiten im öffentlichen Raum - zunächst, da anzunehmen ist, dass der bereits heute steigende Bedarf nach Zu- und Ausstiegsmöglichkeiten mit der Einführung einer automatisierten Tür-zu-Tür-Mobilität weiter steigen wird. Zudem ist es besonders aus kommunaler Sicht bedeutsam, dass automatisierte Mobilitätsdienste vor allem dann einen Beitrag zur Sicherung des öffentlichen Verkehrsnetzes leisten können, wenn durch den Einsatz von Zubringerdiensten die Gesamtreisezeit des ÖPNV reduziert wird (Sinner/Weidmann 2019). Dabei ist die Qualität der Anschlusssicherung nicht nur für die Effizienzsteigerung des Systems elementar, sondern auch, um das Umsteigen für NutzerInnen zu attraktivieren (verkehrsplus 2015).

Da davon auszugehen ist, dass sich die Mobilitätsoptionen aufgrund neuer Technologien und Geschäftsmodelle weiter vervielfältigen, empfiehlt es sich, künftig zwischen unterschiedlichen Formen des temporären Haltens zu differenzieren und Flächen entsprechend zu priorisieren. Eine monomodale Ausrichtung von Straßenzügen und öffentlichen Räumen erscheint vor diesem Hintergrund nicht zeitgemäß, weshalb es an den Kommunen und Planungsämtern liegt, den Wandel vom langfristigen Parken hin zu vielfältigen Nutzungsmöglichkeiten und flexibler Flächenzuweisung einzuläuten.

In Anbetracht des steigenden Nutzungsdrucks werden unterschiedliche Steuerungsweisen diskutiert, um speziell das temporäre Halten im öffentlichen Raum zu regeln. Dabei werden Preisstrategien immer wieder als naheliegende Maßnahme genannt, um unterschiedliche Zwecke zu priorisieren und zeitlich einzuschränken (ITF 2018, NACTO 2019, Marsden et al. 2020). Dies wirft die grundlegende Frage nach der Verwertbarkeit des öffentlichen Raums auf: Das betrifft einerseits den Aspekt, wer zu welchem Zweck Gebühren verlangen darf (vgl. Marsden et al. 
2020: 8), andererseits muss ganz grundsätzlich hinterfragt werden, welche Nutzergruppen von einer straßenräumlichen Veränderung in welchem Ausmaß profitieren. So könnte beispielsweise die reine Umnutzung von straßengebundenen Stellplätzen als Haltezonen zur Folge haben, dass die öffentliche Hand an bedeutsamen Einkünften verlieren würde, währenddessen private Mobilitätsdienstleister von einem kostenfreien Zugriff überdurchschnittlich profitieren (ebd., Mitteregger et al. 2019).

Nachstehend wird in allererster Linie auf gestalterische Maßnahmen eingegangen, die nebst fiskalischen Instrumenten einen wesentlichen Handlungsspielraum für Kommunen und Städte darstellen, öffentliche Räume gleichermaßen zu regulieren und für ganz unterschiedliche Aktivitäten nutzbar zu machen. Dabei werden Betrachtungen zur Qualifizierung räumlicher Schnittstellen vorgestellt, womit jene Orte gemeint sind, an denen unterschiedliche Modi miteinander verknüpft werden, in denen die Raumansprüche neuer Mobilitätsdienste sichtbar werden und die als räumliche Schwellen die Zugänglichkeit umliegender Nachbarschaften beeinflussen.

\subsection{MULTIMODALE ÖFFENTLICHE RÄUME: ENTFLECHTUNG UND BÜNDELUNG VON MODI}

Die Vervielfältigung von Mobilitätsangeboten im Straßenraum stellt die Planung vor die bekannte Herausforderung, Sicherheitsansprüche mit jenen der Aufenthaltsqualität und der ökologischen Nachhaltigkeit (Grünräume, Beschattung, Entsiegelung) zu vereinbaren. Dies als kommunale Gestaltungsaufgabe

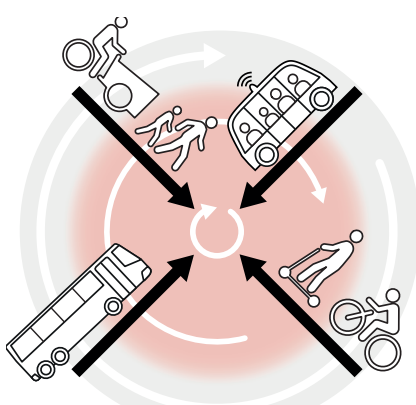
zu begreifen, gewinnt angesichts einer fortschreitenden Digitalisierung und Automatisierung im Mobilitätswesen an Dringlichkeit: zum einen, da die Fahrzeugautomatisierung auf Basis der bestehenden Straßenverkehrsordnung ebenso wie der vorherrschenden Kultur im Straßenverkehr entwickelt wird. Dadurch werden teils tradierte Verhaltensregeln, wie der Vorrang von Modi, die gemeinhin akzeptierten Geschwindigkeiten oder auch die individuelle Fahrzeugnutzung, in künftige Systeme eingeschrieben und durch diese reproduziert. Zum anderen haben Kommunen einen direkten Einfluss auf die Stadtgestalt, wodurch sie auf der Ebene des Straßenraums mittels räumlicher Maßnahmen Geschwindigkeiten, Verkehrsflüsse und Richtungen steuern können (NACTO 2019). Im Sinne multimodaler öffentlicher Räume steht allen voran die Gewährleistung einer sicheren Koexistenz unterschiedlicher Modi. Straßenräume, die bislang durch parkende Autos geprägt waren, sind zugunsten von Fuß- und Radwegen sowie Grünräumen umzunutzen und durch designierte Haltezonen für kurzfristiges Halten auszubauen.

Je nach Straßentypologie und Umfeldnutzungen kann die Errichtung von Haltezonen unterschiedlichen Zwecken dienen (z. B. ÖV-Haltestellen, Zu- und Ausstiege von Mobilitätsdiensten und Privatfahrzeugen, Warenzustellung etc.) und die Gestaltung entsprechend variieren. Die Abstimmung mit den umliegenden Nutzungen ist insbesondere dahingehend erforderlich, als diese unterschiedliche, oftmals tageszeitabhängige Anforderungen an den öffentlichen Raum stellen. Bei der Planung von Standorten und tageszeitabhängigen Regelungen sind demnach neben dem Mobilitätsbedarf besonders die lokalen Aktivitätsspitzen, wie der Schulbeginn am Morgen, zu berücksichtigen. Im Fall von schmalen Straßenbreiten und verkehrsberuhigten Räumen, wie sogenannten „shared spaces“ oder Begegnungszonen, kann das Halten zum Zweck von Ein- und Ausstieg auf der Fahrspur selbst zulässig sein.

Zwar zeigen Studien, dass dies den Verkehrsfluss behindern kann und längere Reisezeiten für automatisierte Mobilitätsdienste zur Folge hätte, allerdings kann sich die geringere Attraktivi- 
tät des motorisierten Verkehrs zugunsten der aktiven Mobilität auswirken (Elvarsson 2017, ITF 2018). Alternativ sind Haltezonen am Straßenrand zu verorten und durch eine Fahrbahnmarkierung oder einen eigenen Bodenbelag zu kennzeichnen. Die Ausweisung fördert die räumliche Bündelung von kurzfristigen Haltevorgängen sowie eine sichere Interaktion mit dem Fuß- und Radverkehr. Im Fall größerer Straßenquerschnitte können auch eigene Haltebuchten errichtet werden, wodurch die Abfahrt von der Fahrbahn erforderlich und der Verkehrsfluss am geringsten beeinträchtigt wäre (ITF 2018: 70). Unter Berücksichtigung des Nutzungsumfelds können Haltebuchten als Teil von Multifunktionsbändern am Straßenrand geplant werden, was die Anbindung an das Fuß- und Radwegenetz sowie die Integration ergänzender Mobilitätsinfrastruktur, von Grünflächen und Aufenthaltsräumen ermöglicht.

Weiters ist bei der Planung von Haltezonen insbesondere auf das bestehende Stationsnetz des ÖPNV zu achten. Um den intermodalen Umstieg und Zugriff auf andere Modi möglichst niederschwellig zu gestalten, bedarf es einer effizienten Flächenanordnung und möglichst kurzen Wegen zwischen Haltezonen automatisierter Mobilitätsdienste, dem ÖPNV und Angeboten der Mikromobilität (z. B. E-Scootern, Lastenrädern etc.). Hierzu sind eine Reihe funktionaler Anforderungen zu berücksichtigen (verkehrplus 2015):

- einfache Orientierung,

- Übersichtlichkeit,

- logische Abfolge der Einrichtungen wie zum Beispiel Ticketautomaten, Wartebereiche und Informationsmöglichkeiten

- mehrere Linien nutzen dieselben Haltestellen oder Haltezonen,

- mehrere Anbieter nutzen dieselben Haltestellen oder Haltezonen,

- Umsteigepunkte liegen knapp beieinander.

Tabelle 5: Multimodale öffentliche Räume: Entflechtung und Bündelung von Modi

\section{Räumlich differenzierte Betrachtung}

\begin{tabular}{ll}
\hline Städtischer Raum & $\begin{array}{l}\text { In dichten Siedlungsgebieten ist der Druck auf öffentliche Räume am höchsten. } \\
\text { Hier gilt es, straßengebundene Stellplätze durch Haltezonen, Räume der Interaktion } \\
\text { sowie Grünflächen zu ersetzen. Die unterschiedlichen Nutzungsansprüche sind } \\
\text { mit dem Umfeld resp. den Aktivitäten abzustimmen und Haltezonen bevorzugt an } \\
\text { bestehenden Stationen des ÖPNV zu integrieren. }\end{array}$ \\
\hline Suburbaner Raum & $\begin{array}{l}\text { In suburbanen Gebieten ist bei der Planung von Haltezonen für automatisierte } \\
\text { Mobilitätsdienste auch der Ausbau der Radverkehrsinfrastruktur zu berücksichtigen. } \\
\text { Beide Verkehrsmittel können eine wichtige Zubringerfunktion für den höherrangigen } \\
\text { ÖPNV erfüllen und erfordern entsprechende Infrastrukturvorkehrungen und } \\
\text { Sichtbarkeit. }\end{array}$ \\
\hline Ländlicher Raum & $\begin{array}{l}\text { In ländlichen Räumen ist bei der Einrichtung von Haltepunkten für automatisierte } \\
\text { Mobilitätsdienste zu berücksichtigen, dass diese als Teil von zusammenhängenden } \\
\text { Fuß- und Radwegen geplant werden und als prägnanter Aufenthaltsort sichtbar } \\
\text { gemacht werden. }\end{array}$ \\
\hline
\end{tabular}




\subsection{FLEXIBLE FLÄCHENDIMENSIONIERUNG: BEDARFSAB- GESTIMMT ODER TAGESZEITABHÄNGIG}

Für die Ermittlung eines angemessenen Flächenausmaßes von Haltezonen ist eine Bedarfsmodellierung erforderlich. Diese kann beispielsweise auf Basis von Aktivitätsmustern, d. h. den Verkehrserzeugungsraten umliegender Nutzungen und dem erwarteten Mobilitätsverhalten im Verhältnis zu strukturellen Parametern (z. B. Einwohner- und Beschäftigungsdichte, Erreichbar-

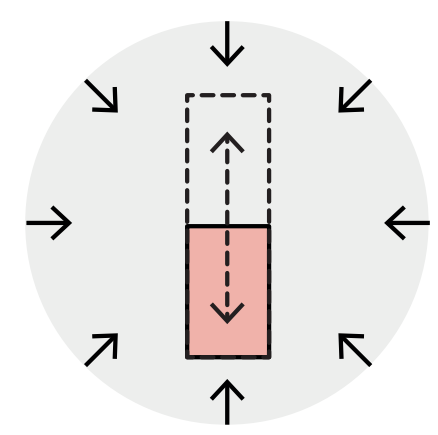
keit des ÖPNV, Modal Split etc.) des jeweiligen Einzugsgebiets bestimmt werden (Schwartz 2017, Bruns et al. 2018). Allerdings ist anzunehmen, dass die Fahrzeugautomatisierung zu einer Mobilisierung bisher mobilitätseingeschränkter Personengruppen beitragen wird und dadurch einen latenten Anstieg des Verkehrsaufkommens verursachen kann. Grundannahmen, wie die, dass eine Haltefläche pro Stunde bis zu $80 \mathrm{Zu}$ - und Ausstiege bedienen kann, wenn von einer durchschnittlichen Zu- und Ausstiegsdauer von 45 Sekunden ausgegangen wird (ITF 2018: 66), sind durch lokale Bedarfserhebungen zu verifizieren und anzupassen. Auch kann die Flächendimensionierung je nach Verortung entlang eines Straßenzuges unterschiedlich behandelt werden. Im Fall von Haltebuchten sind beispielsweise ergänzende Flächen für die Zu- und Einfahrt zu berücksichtigen, die allerdings bei einer flächenschonenden Verortung vor oder nach Kreuzungsräumen geringer ausfallen (Fehr \& Peers 2019: 19).

Wie Studien zu den Parkraumanforderungen automatisierter Angebotsformen zeigen konnten, sind neben Haltezonen für das Zu- und Aussteigen auch Flächenerfordernisse für kürzere Wartezeiten zwischen einzelnen Fahrten zu berücksichtigen (Kondor et al. 2020: 17). Besonders wenn ein erhöhtes Ausmaß an Leerfahrten vermieden werden soll, können diese einen ausschlaggebenden Anteil des künftigen Parkraumbedarfs automatisierter Flotten ausmachen (ebd.). Zu berücksichtigen ist, dass das erforderliche Flächenausmaß je nach Siedlungs- und Nachfragedichte und maximaler Wartezeit für Fahrgäste ansteigen kann, da auch die Anzahl zeitgleich wartender Fahrzeuge zunehmen könnte. Dafür ist unter anderem die Standortdichte von Haltezonen sowie das Einzugsgebiet von Mobilitätsflotten ausschlaggebend (ebd.). Beide Faktoren können durch unterschiedliche Maßnahmen der öffentlichen Hand, wie durch die Festlegung von Bedienungsgebieten, Nutzungsgebühren oder einer maximalen Anzahl an nutzbaren Stellplatzflächen, gesteuert werden.

Darüber hinaus ist auch zu berücksichtigen, dass der Flächenbedarf je nach Tageszeit und Netzwerkkapazität variieren kann. Bei entsprechender Datengrundlage und Echtzeiterhebung könnten Haltezonen künftig dynamisch ausgewiesen und die Nutzung des öffentlichen Raums außerhalb von Stoßzeiten dem Aufenthalt und der aktiven Mobilität gewidmet werden. Davon würden besonders Orte hoher Nutzungsdichte profitieren, wie zum Beispiel Schulen, Bürobauten oder Kulturstätten, in deren Umkreis temporäre Erweiterungsflächen zum Zeitpunkt von Aktivitätsspitzen Abhilfe leisten können (Fehr \& Peers 2019). Auch Halteflächen für Lieferverkehre, mobile Dienste oder die Außengastronomie (Food Trucks) können auf dezidierte Tages- und Nachtzeiten beschränkt werden, um benötigte Flächen möglichst ressourcenschonend zur Verfügung zu stellen (NACTO 2019: 120). Dabei kann der Zugriff auf Haltezonen durch Nutzungsgebühren geregelt werden, die je nach Nachfrage, Nutzungsart und Besetzungsraten sowie Antriebsart variabel gestaltet werden können.

Zu guter Letzt hängt der potentielle Rückgewinn öffentlicher Räume von der Priorisierung der Flächenansprüche ab. Städte und Kommunen haben zu klären, welchen Mobilitätsarten und Nutzungsgruppen künftig Vorrang gewährt wird und inwieweit neben ÖPNV-Haltestellen auch das ungehinderte Halten von Krankentransporten oder Fahrtendiensten für mobilitätseingeschränkte Personen Vorrang erhält. Hinsichtlich einer flächendeckenden Umnutzung von Stellplätzen wird 
nicht zuletzt die öffentliche Akzeptanz entscheidend sein. Künftige Planungsprozesse haben dahingehend besonders die Aushandlung von Nutzungsansprüchen zwischen öffentlichen und privaten Mobilitätsanbietern, Anrainern und lokalen Gewerbetreibenden zu berücksichtigen.

Tabelle 6: Flexible Flächendimensionierung: bedarfsabgestimmt oder tageszeitabhängig

\begin{tabular}{ll}
\hline \multicolumn{1}{c}{ Räumlich differenzierte Betrachtung } \\
\hline Städtischer Raum & $\begin{array}{l}\text { Besonders in innerstädtischen Gebieten könnten dynamische Haltezonen, die auf } \\
\text { Basis von Echtzeitdaten ausgewiesen werden, an Orten hoher Nutzungsdichte sowie } \\
\text { vor/nach Großveranstaltungen als Ergänzung zu statischen Flächen Abhilfe leisten. }\end{array}$ \\
\hline Suburbaner Raum & $\begin{array}{l}\text { Aufgrund geringerer Nutzungsdichten ist anzunehmen, dass in suburbanen Gebieten } \\
\text { die Bereitstellung statischer Haltezonen genügt. Allerdings ist auch in diesen } \\
\text { Gebieten eine tageszeitliche Priorisierung mit den jeweiligen Umfeldnutzungen } \\
\text { abzustimmen. }\end{array}$ \\
\hline Ländlicher Raum & $\begin{array}{l}\text { Aufgrund der geringen Dichte ländlicher Räume ist mit geringen } \\
\text { Futzungen (z.B. Fahrgastinformation, Abstellmöglichkeiten, Paketzustellung etc.) } \\
\text { standardmäßig mehr Flächen zugewiesen werden können. }\end{array}$ \\
\hline
\end{tabular}

Quelle: eigene Darstellung

\subsection{SANFTE ÜBERGÄNGE: DURCHLÄSSIG UND ZUM VERWEILEN EINLADEND}

In Anbetracht möglicher Flächeninanspruchnahme und räumlicher Trennwirkungen, die das automatisierte Fahren zur Folge haben könnte (siehe Beitrag 5 von Soteropoulos in diesem Band), bedarf es konkreter Gestaltungsansätze, um die Durchlässigkeit und Aufenthaltsqualität von öffentlichen Räumen künftig zu gewährleisten. Die Gestaltung „sanfter Übergänge“ (Gehl 1971: 183), wonach ein situationsangemessenes Verhältnis zwischen Ordnung (im Sinne der Verkehrssicherheit) und Freiheit (im Sinne des ungeregelten Aufenthalts und der subjektiven Aneignung) anzustreben ist, stellt eine zentrale Aufgabe der Stadtplanung dar (vgl. Bormann 2014).

Kleinräumig betrifft dies zunächst das Querungsbedürfnis von FußgängerInnen, das im Verhältnis zur Aktivitätsdichte der Nutzungsnachbarschaft - Konzentration von Handel, Gastronomie oder öffentlichen Einrichtungen - steht. Um eine sichere Interaktion mit dem Fuß- oder Radverkehr zu ermöglichen, sind je nach Kontextbedingungen und Lage im Verkehrsnetz möglichst geringe Geschwindigkeiten für den motorisierten Verkehr festzulegen. Zudem können der jeweiligen Straßentypologie entsprechende Maßnahmen, wie schmälere Fahrbahnbreiten, flexible Verkehrsinseln und Mehrzweckstreifen oder unterschiedliche Oberflächenbeläge, dazu beitragen, dass Straßenräume durchquerbar bleiben und Sichträume geschaffen werden (Larco/Tierney 2020, NACTO 2019). Konsequenterweise bedeutet dies für die Gestaltung von Haltezonen oder Haltebuchten, dass auch diese von mehreren Seiten barrierefrei zugänglich zu planen sind. Nicht zuletzt begünstigen flächige Querungsmöglichkeiten im Sinne von „shared spaces“ die Durchlässigkeit eines öffentlichen Raums; sie eignen sich besonders innerörtlich und in Räumen mit hoher Zentrumsqualität, die in der Regel einen überdurchschnittlichen Querungsbedarf aufweisen (Ghielmetti et al. 2017). Weitere Faktoren, die eine Errichtung von „shared spaces“ begünstigen, inkludieren das Vorliegen geringer Straßenquerschnitte und 
niedriger Verkehrsmengen. Ist die fußläufige Durchlässigkeit des öffentlichen Raums gewährleistet, kann sich dies auf mehrere Arten positiv auswirken: kürzere Wege für Zufußgehende, erhöhte Fußgängerfrequenz entlang umliegender Erdgeschossnutzungen, höhere Stetigkeit des fahrenden Verkehrs sowie das Ausbleiben negativer Auswirkungen auf die Verkehrssicherheit (vgl. Ghielmetti et al. 2017: 14).

Um räumliche Schnittstellen nicht auf funktionale Transitorte zu reduzieren, sind diese ebenso als Räume des Aufenthalts und der Interaktion zu verstehen. Hierfür bedarf es neben der räumlichen Zugänglichkeit im Sinne direkter Zu- und Abwege, auch der Ausgestaltung von attraktiven Rastplätzen, an denen Begegnungsmöglichkeiten entstehen können. Folgt man dem Diskurs der IT- und Automobil-Industrie, so dient die digitale Vernetzung und Automatisierung des Mobilitätswesens unter anderem der Einrichtung schnittstellenloser Mobilität (,seamless mobility“). Dies beschreibt neben der Integration operativer Prozesse zur Bedienungserleichterung auch ein Zukunftsbild möglichst komfortabler Tür-zu-Tür-Mobilität.

Wird der öffentliche Raum jedoch rein auf ein schnelles Kommen und Gehen ausgerichtet, so würden jene länger andauernden Aktivitäten verschwinden, die wesentlich zur Lebendigkeit des öffentlichen Raums beitragen (Gehl 1971). Sanfte Übergänge meint demnach auch ein Abschwächen der Effizienzlogik und der Entschleunigung, sodass im Umfeld von Haltezonen und Sammelpunkten unterschiedliche Aktivitäten zeitgleich stattfinden können: warten, sitzen, ausleihen, buchen, abholen/aufgeben, reparieren, informieren, lernen, austauschen, treffen, erfrischen. Die Haltezone oder der Sammelpunkt dient so nicht nur als Knoten im Verkehrsnetz, sondern auch als ein sozialer Begegnungsraum (Soike et al. 2019).

Inwieweit soziale Ansprüche des Aufenthalts und der Kommunikation berücksichtigt werden, wirkt sich letztlich auf die Lebensqualität im öffentlichen Raum aus (vgl. Weidmann et al. 2011). Im Hinblick auf die mit der Digitalisierung einhergehende Tendenz der Enträumlichung gewinnt dieser Aspekt an Bedeutung. Entgegen mancher Befürchtung, die Lebendigkeit öffentlicher Räume könnte aufgrund des Internet an Stellenwert verlieren, machen es gerade Phasen technologischer Beschleunigung, wie die durch die Covid-19-Pandemie ausgelöste Ausnahmesituation im Frühjahr 2020, deutlich, dass die soziale Interaktion und die Bewegung im gebauten Raum einen elementaren Ausgleich zur Navigation digitaler Sphären darstellt (Banerjee 2001). Damit steigt nicht zuletzt die Bedeutung der Gestaltungsqualität, die bestenfalls zu einem Verweilen, sicheren Spiel und Austausch im Freien einlädt.

Tabelle 7: Sanfte Übergänge: durchlässig und zum Verweilen einladend

\section{Räumlich differenzierte Betrachtung}

\begin{tabular}{ll}
\hline Städtischer Raum & $\begin{array}{l}\text { In dichten Siedlungsstrukturen kommt der Durchlässigkeit öffentlicher Räume die } \\
\text { größte Bedeutung zu. Der Erhalt von nachbarschaftlichen Beziehungen und hoher } \\
\text { Aufenthaltsqualität von öffentlichen Räumen ist in Anbetracht einer zunehmenden } \\
\text { Mensch-Maschine-Interaktion zu priorisieren. }\end{array}$ \\
\hline Suburbaner Raum & $\begin{array}{l}\text { Auch in suburbanen Gebieten empfiehlt es sich, öffentliche Räume im Sinne } \\
\text { der fußläufigen Querung als „shared spaces“ aufzuwerten. Bei geringen } \\
\text { Geschwindigkeiten des automatisierten Fahrens und ebenerdiger Gestaltung kann } \\
\text { die Nutzungsvielfalt und Aufenthaltsqualität gefördert werden. }\end{array}$ \\
\hline Ländlicher Raum & $\begin{array}{l}\text { Besonders in ländlichen Räumen zählt die Gestaltungsqualität von öffentlichen Räumen } \\
\text { zu einem Schlüsselelement der Ortskernbelebung. Fließende Übergänge zwischen } \\
\text { Fahrbahn und umliegenden Nutzungen, straßenbündige Haltezonen und integrierte } \\
\text { Verweilmöglichkeiten können zu einem Zentrumscharakter wesentlich beitragen. }\end{array}$ \\
\hline
\end{tabular}




\subsection{AUSSTATTUNG UND ADRESSBILDUNG: IM WECHSELSPIEL MIT DEM UMFELD}

Je nach Standort, Nutzungsansprüchen und Flächenverfügbarkeit sind an räumlichen Schnittstellen unterschiedliche Ausstattungselemente zu integrieren. Dazu können Haltezonen durch eine Reihe von baulichen Elementen ergänzt werden, so etwa durch Sitzgelegenheiten, eine Überdachung, Licht- und Informationssäulen, Abstellplätze für Fahrräder und Scooter sowie

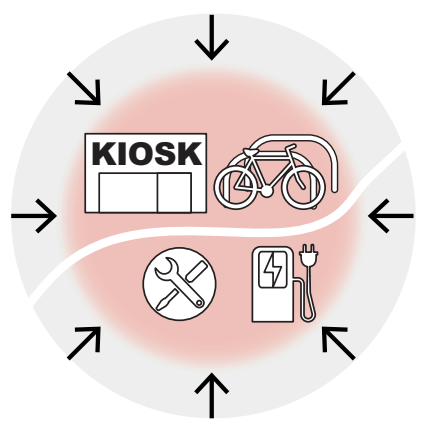
Ladesäulen für E-Fahrzeuge. Eine modulare Gestaltung der Ausstattungselemente kann dabei die langfristige Adaptivität begünstigen (Lehmann 2011). Wesentlich ist, dass die Bündelung von Mobilitätsangeboten zu einer Ordnung des ruhenden Verkehrs und einer Verbesserung der Aufenthaltsqualität beitragen kann. Um die öffentliche Sichtbarkeit und die Akzeptanz der Mobilitätsangebote positiv zu beeinflussen, setzen bestehende Konzepte multimodaler Mobilitätspunkte zudem auf eine einheitliche Kennzeichnung durch eine Farbgebung und Beschilderung von Stationen (Schlump et al. 2014: 81, Stadt Wien 2018). Auch im Fall einer Errichtung von dezidierten Haltezonen und Sammelpunkten für automatisierte Angebotsformen wird ebenso auf eine attraktive Ausstattung und Adressbildung zu achten sein, um die öffentliche Wahrnehmung und Nutzerakzeptanz zu fördern.

Für die Bewertung der Angemessenheit und die bauliche Integration von „funktionsunterstützender Infrastruktur" (Schlump et al. 2014: 10) ist die Abstimmung mit dem direkten Umfeld entscheidend. So sind für eine gezielte Ausstattungswahl angrenzende öffentliche Räume (z. B. Parks, Plätze, Märkte etc.) und die Erdgeschossnutzungen der umliegenden Bebauung (z. B. Wohnen, Bildung, Gewerbe, Tourismus etc.) zu berücksichtigen. Besonders innerorts dienen Fußwege nicht lediglich der aktiven Mobilität oder dem Zustieg auf andere Modi, sondern vermitteln als öffentlicher Raum ebenso zwischen den angrenzenden Erdgeschosszonen. Wie schon Jane Jacobs konstatierte (1961), wird der öffentliche Raum durch die Öffentlichkeit begleitender Nutzungen belebt (z. B. Gewerbe, Serviceeinrichtungen, Gastronomie, Kulturstätten etc.). Dabei kann ein positives Verhältnis von innen und außen sowohl das Sicherheitsgefühl als auch die Aktivitätsvielfalt von öffentlichen Räumen beeinflussen. Bauliche Elemente sind demnach so im öffentlichen Raum zu verorten, dass sie zu dessen Übersichtlichkeit beitragen und die Durchlässigkeit für den Fuß- und Radverkehr fördern (Ghielmetti et al. 2017).

Tabelle 8: Ausstattung und Adressbildung: im Wechselspiel mit dem Umfeld

\section{Räumlich differenzierte Betrachtung}

\begin{tabular}{ll}
\hline Städtischer Raum & $\begin{array}{l}\text { Besonders in innerstädtischen Gebieten besteht die Herausforderung, heterogene } \\
\text { Nutzungen im öffentlichen Raum zu integrieren. Die hohe Siedlungsdichte legt nahe, } \\
\text { dass Funktionen wie E-Ladesäulen oder Paketstationen in angrenzende Erdgeschosse } \\
\text { ausgelagert werden, sodass straßenräumliche Flächen entlastet werden. }\end{array}$ \\
\hline \multirow{2}{*}{ Suburbaner Raum } & $\begin{array}{l}\text { In suburbanen Räumen besteht das Potential, durch die Nutzungsanreicherung von } \\
\text { Treffpunkte zu schaffen, so diese durch eine räumliche Gestaltung angemessen } \\
\text { erfahrbar gemacht werden. }\end{array}$ \\
\hline Ländlicher Raum & $\begin{array}{l}\text { Auch ländliche Räume können von einer erhöhten Sichtbarkeit von Sammelpunkten } \\
\text { profitieren, wobei besonders baukulturelle Akzente als Träger einer lokalen Identität } \\
\text { dienen können (Kaltenbach 2013). Diese wirken nicht nur landschaftsbildprägend, } \\
\text { sondern können auch touristische Aktivität begünstigen und somit stabilisierend wirken. }\end{array}$ \\
\hline
\end{tabular}


Im Sinne einer Entlastung öffentlicher Räume ist auch zu prüfen, inwieweit angrenzende Erdgeschosszonen als Bestandteil von Mobilitätspunkten bespielt werden können. Besonders straßenraumorientierte Garagenflächen oder Leerstände würden sich für mobilitätsfördernde Zwecke wie E-Ladestationen, Reparaturwerkstätten oder Verleihdienste anbieten. Ein unmittelbarer Zugang ermöglicht, dass Aktivitäten immer wieder auch in den öffentlichen Raum hinausfließen und inn bereichern. Öffentliche Räume könnten so von den Flächenanforderungen neuer Mobilitäts- und Lieferkonzepte freigehalten und stattdessen nutzungsoffen gestaltet werden.

\section{RESÜMEE UND AUSBLICK}

Auch unter den Vorzeichen des automatisierten Fahrens ist die Lebensqualität öffentlicher Räume das Ergebnis eines Gestaltungsprozesses, in dessen Rahmen die Einsatzbereiche und Ziele neuer Mobilitätskonzepte bestimmt werden (vgl. Weidmann et al. 2011). Die fortschreitende Heterogenisierung der Mobilitätsbedürfnisse und die Dringlichkeit, auf Klima- und Gesundheitskrisen zu reagieren, machen deutlich, dass die angemessene Berücksichtigung teils widersprüchlicher Nutzungsansprüche im öffentlichen Raum eine zentrale Herausforderung für die Stadtplanung darstellt. Die Übergangsphase hin zu einer automatisierten Mobilität verleiht diesem Gestaltungsauftrag zusätzliche Komplexität: einerseits, da der Verlauf der tatsächlichen technologischen Einführung bis auf Weiteres unklar bleibt, und andererseits, da die räumlichen Wirkungen der Übergangsphase (z. B. Veränderungen im Parkraumbedarf) kaum anhand von Szenarien des Mischverkehrs, in denen sich zufußgehende Personen, Fahrradfahrende und (automatisierte) Fahrzeuge den Straßenraum teilen, erforscht worden sind (Zhang/Wang 2020). Dennoch bietet die frühzeitige Auseinandersetzung mit möglichen Wirkungen des automatisierten Fahrens die Chance, Planungsprinzipien und Flächenansprüche, die der aktuellen Entwicklung öffentlicher Räume zugrunde liegen und im Begriff stehen, den künftigen Verlauf der automatisierten Mobilität zu prägen, in Frage zu stellen und neu zu bewerten.

Die hier dargelegten Betrachtungen machen deutlich, dass die Stadtplanung künftig umso mehr gefordert sein wird, die Steuerungsansätze des Verkehrsmanagements mit den Steuerungs- und Gestaltungsansätzen der Stadtentwicklung abzustimmen und deren Wechselwirkungen zu berücksichtigen. Es ist dabei unerlässlich, die Entwicklungen im Mobilitätswesen aus der Perspektive des öffentlichen Raums heraus zu überprüfen, da die Gestaltungsqualität räumlicher Schnittstellen auf Mobilitätsverhalten, Stadtgestalt und Siedlungsentwicklung wirkt - zumal das Ausmaß an räumlicher Veränderung je nach Stadtraum, Dichte, Verkehrsaufkommen und Zugänglichkeit variieren kann (Larco/Tierney 2020). Hierzu bedarf es im Rahmen weiterer Auseinandersetzungen und Forschung einer räumlichen Differenzierung, beispielsweise anhand von Stadtraumtypen und Straßentypologien sowie anhand von konkreten räumlichen Fallbeispielen im städtischen bis ländlichen Raum.

Perspektivisch bedarf es in Anbetracht des automatisierten Fahrens einer strategischen Festlegung von Einsatzgebieten sowie einer räumlich differenzierten Zulassung von Modi und Geschwindigkeiten. Die Stadtplanung hat dahingehend die wesentliche Aufgabe, mögliche Ansätze durch eine Beurteilung der Stadtverträglichkeit und des räumlichen Mehrwerts zu schärfen. Zudem bedarf es einer öffentlichen Auseinandersetzung mit der Frage, wie der Weg hin zu einer flexiblen und temporären Nutzung öffentlicher Räume zu gestalten ist. Inwieweit kann die Bereitstellung von Infrastruktur für geteilte Mobilitätsdienste und künftig automatisierte Verkehre mittels Pilotvorhaben schon heute erprobt werden? Welche Nutzungsarten 
sind vor dem Hintergrund einer steigenden Vielfalt an Flächenansprüchen zu priorisieren? Unter welchen Bedingungen kann eine dynamische Nutzungsverteilung von Vorteil sein? Und in welchem Ausmaß kann die Thematik in vorhandene Planungsstrategien und Konzepte integriert werden, sodass mittelfristige Vorhaben mit bestehenden Entwicklungsrichtungen abgeglichen werden? Die kommunale Aufgabe besteht darin, durch die Abstimmung unterschiedlicher Zeithorizonte und durch die Balance von Offenheit und einer gezielten Steuerung öffentliche Räume mit automatisiertem Fahren als vielfältig nutzbare und wandlungsfähige Lebensräume zu stärken.

\section{LITERATUR}

Angélil, M., K. Christiaanse, V. M. Lampugnani, C. Schmid und G. Vogt 2012. „Urbane Potenziale und Strategien in metropolitanen Territorien. Am Beispiel des Metropolitanraums Zürich“, Nationales Forschungsprogramm NFP65 - Neue urbane Qualität, ETH Zürich: Departement Architektur. https://www.christiaanse.arch.ethz.ch/upload/up.pdf (14.5.2020).

ARE (Bundesamt für Raumentwicklung) 2004. „Räumliche Auswirkungen der Zürcher S-Bahn - eine expost Analyse. Zusammenfassung“. Bern: UVEK Eidgenössisches Departement für Umwelt, Verkehr, Energie und Kommunikation. Download unter https://www.are.admin.ch/are/de/home/mobilitaet/ programme-und-projekte/raeumliche-auswirkungen-der-verkehrsinfrastrukturen/raeumliche-auswirkungen-der-zuercher-s-bahn.html (20.10.2020).

Arndt, W.-H., und F. Drews 2019. „Mobilität nachhaltig planen. Erfolge und Hindernisse in deutschen Städten - Ergebnisse einer Umfrage zu kommunalen Verkehrsentwicklungsplänen“. Sonderveröffentlichungen. Deutsches Institut für Urbanistik. https://difu.de/publikationen/2019/mobilitaetnachhaltig-planen (14.5.2020).

Backhaus, W., S. Rupprecht und D. Franco 2019. „Practitioner Briefing: Road vehicle automation in sustainable urban mobility planning“, hg. v. Rupprecht Consult - Forschung \& Beratung. https:// www.eltis.org/sites/default/files/road_vehicle_automation_in_sustainable_urban_mobility_planning_0.pdf (14.5.2020).

Banerjee, T. 2001. „The future of public space: Beyond invented streets and reinvented places“, in Journal of the American Planning Association (67) 1, 9-24. DOI: 10.1080/01944360108976352.

Banister, D. 2008. „The sustainable mobility paradigm“, in Transport Policy (15) 2, 73-80.

Beckmann, K. D., J. Gies, J. Thiemann-Linden und T. Preuß 2011. „Leitkonzept - Stadt und Region der kurzen Wege. Gutachten im Kontext der Biodiversitätsstrategie“, Texte 48, Sachverständigengutachten. Dessau-Roßlau: Bundesministerium für Umwelt, Naturschutz und Reaktorsicherheit. www. umweltbundesamt.de/sites/default/files/medien/461/publikationen/4151.pdf (14.5.2020).

Bendiks, S., und A. Degros 2019. Traffic Space is Public Space. A Handbook for Transformation, 1. Aufl. Zürich: Park Books .

Bertolini, L. 1999. „Spatial Development Patterns and Public Transport: The Application of an Analytical Model in the Netherlands“, in Planning Practice and Research 14, 199-210. DOI: 10.1080/02697459915724.

Bienzeisler, B., S. Bengel, M. Handrich und S. Martinetz 2019. „Die digitale Transformation des städtischen Parkens. Eine Analyse der Veränderung des kommunalen Parkraummanagements vor dem Hintergrund der Herausforderungen einer Verkehrswende“. Stuttgart: Fraunhofer IAO - Institut für Arbeitswirtschaft und Organisation. http://publica.fraunhofer.de/eprints/urn_nbn_de_0011n-5381331.pdf (14.5.2020).

Bormann, O. 2014. „Aktuelle Verkehrslage - Von der Rückgewinnung urbaner Infrastruktur“, in Architektur im Kontext, hg. v. K. von Keitz und S. Voggenreiter. Berlin: jovis, 96-109.

Bremer, S. 2017. „Kommunale Mobilitätspläne und ihre Umsetzung“, Vortrag, Zukunftsnetzwerk Mobilität NRW. 16.1.2017. ttps://mobilitaetsmanagement.nrw.de/sites/default/files/downloads/05_bremer_mobilitaetsplaene.pdf (14.5.2020). 
Bruck, E. M. 2019. „Automatisierte Mobilitätsdienste als Wandlungsimpuls für suburbane Räume?“, in Broadacre City 2.0 - postfossil. Ein urbanistisches Szenario für 2050, hg. v. J. Fiedler. Graz: Haus der Architektur.

Bruns, F., B. Tasnady, N. de Vries, N. Frischknecht, E. Selz, S. Grössl und M. Berger 2018. „Verfahren und Kennwerte zur Abschätzung von Verkehrswirkungen“, Forschungsprojekt SVI 2014/005 auf Antrag der Schweizerischen Vereinigung der Verkehrsingenieure und Verkehrsexperten (SVI). Bern: Eidgenössisches Departement für Umwelt, Verkehr, Energie und Kommunikation UVEK. http://www. yverkehrsplanung.at/images/ProjektauswahI/SVI_2014_005_Schlussbericht_2018-09-18.pdf (22.10.2020).

Cavoli, C., B. Phillips, T. Cohen und P. Jones 2017. „Social and behavioural questions associated with Automated Vehicles. A Literature Review“. London: UCL - University College, Department for Transport. https://www.ucl.ac.uk/transport/sites/transport/files/social-and-behavioural-literaturereview.pdf (14.5.2020).

Cohen, T., und C. Cavoli 2019. „Automated vehicles: Exploring possible consequences of government (non)intervention for congestion and accessibility“, in Transport Reviews (39) 1, 129-151.

Elvarsson, A. B. 2017. „Modelling Urban Driving and Stopping Behavior for Automated Vehicles, Semester Project“. Zürich: ETH, IVT - Institut für Verkehrsplanung und Transportsysteme. https://ethz. ch/content/dam/ethz/special-interest/baug/ivt/ivt-dam/publications/students/501-600/sa597.pdf (15.10.2020).

Erhardt, G. D., S. Roy, D. Cooper, B. Sana, M. Chen und J. Castiglione 2019. „Do transportation network companies decrease or increase congestion?“, in Science Advances (5) 5, eaau2670. DOI: 10.1126/ sciadv.aau2670.

Fehr \& Peers 2019. „Cincinnati Curb Study“. www.fehrandpeers.com/curbs-of-the-future/ (14.5.2020).

Gavanas, N. 2019. „Autonomous Road Vehicles: Challenges for Urban Planning in European Cities“, in Urban Science (3) 2, 61. DOI: 10.3390/urbansci3020061.

Gehl, J. 2012. Leben zwischen Häusern. Konzepte für den öffentlichen Raum. Berlin: jovis.

Ghielmetti, M., R. Steiner, J. Leitner, M. Hackenfort, S. Diener und H. Topp 2017. „Flächiges Queren in Ortszentren - Langfristige Wirkung und Zweckmässigkeit“, Forschungsprojekt SVI 2011/023 auf Antrag der Schweizerischen Vereinigung der Verkehrsingenieure und Verkehrsexperten (SVI). Bern: Eidgenössisches Departement für Umwelt, Verkehr, Energie und Kommunikation UVEK. Download unter duct/24100/?q=fl\%C3\%A4chiges\%20queren\&tx_solr\%5Bfilter\%5D\%5B0\%5D=facet_212_stringM\%253AAktiv\&tx_solr\%5Bpage\%5D=0\&cHash=6b09fe1968d32b1011e3865d5564cc4a (14.5.2020).

González-González, E., S. Nogués und D. Stead 2020. „Parking futures: Preparing European cities for the advent of automated vehicles“, in Land Use Policy 91, 104010. DOI: 10.1016/j.landusepol.2019.05.029.

Graehler, M., R. A. Mucci und G. D. Erhardt 2019. „Understanding the Recent Transit Ridership Decline in Major US Cities: Service Cuts or Emerging Modes?" Conference: Transportation Research Board Annual Meeting, Washington, DC. https://www.researchgate.net/publication/330599129_Understanding_the_Recent_Transit_Ridership_Decline_in_Major_US_Cities_Service_Cuts_or_Emerging_Modes (14.5.2020).

Greenblatt, J. B., und S. Shaheen 2015. „Automated Vehicles, On-Demand Mobility, and Environmental Impacts“, in Current Sustainable/Renewable Energy Reports (2) 3, 74-81. Download unter https:// link.springer.com/article/10.1007/s40518-015-0038-5 (14.10.2020).

Groth, S. 2019. „Multioptionalität: Ein neuer (,alter`) Terminus in der Alltagsmobilität der modernen Gesellschaft?", in Raumforschung und Raumordnung / Spatial Research and Planning (77) 1, 17-34.

Herget, M., F. Hunsicker, J. Koch, B. Chlond, C.Minster und T. Soylu 2019. „Ökologische und ökonomische Potenziale von Mobilitätskonzepten in Klein- und Mittelzentren sowie dem ländlichen Raum vor dem Hintergrund des demographischen Wandels“, Texte 14. Dessau-Roßlau: Umweltbundesamt. https://www.umweltbundesamt.de/en/publikationen/oekologische-oekonomische-potenzialevon (14.5.2020).

Hörl, S., F. Becker, T. Dubernet und K. W. Axhausen 2019. „Induzierter Verkehr durch autonome Fahrzeuge: Eine Abschätzung“, Forschungsprojekt SVI 2016/001 auf Antrag der Schweizerischen Vereinigung der Verkehrsingenieure und Verkehrsexperten (SVI). Bern: Eidgenössisches Departement für Umwelt, Verkehr, Energie und Kommunikation UVEK. https:/ethz.ch/content/dam/ethz/ special-interest/baug/ivt/ivt-dam/vpl/reports/1401-1500/ab1433.pdf (18.8.2020). 
ITF (International Transport Forum) 2018. „The Shared-Use City: Managing the Curb“, Corporate Partnership Board Report. https://www.itf-oecd.org/sites/default/files/docs/shared-use-city-managing-curb_3.pdf (14.5.2020).

Jacobs, J. 1961. The Death and Life of Great American Cities. New York: Random House.

Jin, S. T., H. Kong, R. Wu und D. Z. Sui 2018. „Ridesourcing, the sharing economy, and the future of cities“, in Cities 76, 96-104.

Jürgens, L. 2020. „Konnektivitätsveränderungen im ÖPNV-Netz durch die Einführung eines autonomen Shuttlebusses", in Autonome Shuttlebusse im ÖPNV: Analysen und Bewertungen zum Fallbeispiel Bad Birnbach aus technischer, gesellschaftlicher und planerischer Sicht, hg. v. A. Riener, A. Appel, W. Dorner, T. Huber, J. C. Kolb und H. Wagner. Berlin/Heidelberg: Springer Vieweg, 39-54.

Kaltenbach, F. 2013. „Typologie: Vom Verkehrsknoten zur Drehscheibe - Haltestellen für integrierte Mobilitätskonzepte“, DETAIL 9. Download unter https://inspiration.detail.de/typologie-vom-verkehrsknoten-zur-drehscheibe-haltestellen-fuer-integrierte-mobilitaetskonzepte-107171.html?lang=en (14.5.2020).

Kondor, D., P. Santi, D.-T. Le, X. Zhang, A. Millard-Ball und C. Ratti 2020. „Addressing the ,minimum parking" problem for on-demand mobility". https://arxiv.org/pdf/1808.05935.pdf (14.10.2020).

Konrad, K., und S. Groth 2019. „Consistency or contradiction? Mobility-Related Attitudes and Travel Mode Use of the Young New Generation“ in Raumforschung und Raumordnung I Spatial Research and Planning (77) 6, 1-17.

Kretz, S., und L. Kueng (Hrsg.). 2016. Urbane Qualitäten - Ein Handbuch am Beispiel der Metropolitanregion Zürich. Zürich: Edition Hochparterre.

Larco, N., und G. Tierney 2020. „Impacts on Urban Design“, in Multilevel Impacts of Emerging Technologies on City Form and Development, hg. v. Howell, A., H. Tan, A. Brown, M. Schlossberg, J. KarlinResnick, R. Lewis, M. Anderson, N. Larco, G. Tierney, J. Carlton, J. Kim und B. Steckler. Eugene, OR: Urbanism Next Center, University of Oregon, 115-141. https://cpb-us-e1.wpmucdn.com/blogs.uoregon.edu/dist/f/13615/files/2020/01/NSF-Report_All-Chapters_FINAL_013020.pdf (15.10.2020).

Lehmann, T. 2011. „Der Bahnhof der Zukunft - Alternativen zum traditionellen Bahnhofsempfangsgebäude I Entwicklung eines modularen Entréesystems für kleine und mittlere Bahnhöfe“, Doktorarbeit, TU Berlin, Fakultät VI - Planen Bauen Umwelt. DOI: 10.14279/depositonce-2920.

Lenz, B., und E. Fraedrich 2015. „Neue Mobilitätskonzepte und autonomes Fahren: Potenziale der Veränderung", in Autonomes Fahren: Technische, rechtliche und gesellschaftliche Aspekte, hg. v. M. Maurer, J. C. Gerdes, B. Lenz und H. Winner. Berlin/Heidelberg: Springer Vieweg, 175-195.

Leszczynski, A., und R. Kitchin 2019. „UBER. The Seduction of UberCity”, in How to Run a City like Amazon, and Other Fables, hg. v. M. Graham, R. Kitchin, S. Mattern und J. Shaw. London: Meatspace Press, 1179-1195.

Lewis, R., und M. Anderson 2020. „Impacts on Land Use“, in Multilevel Impacts of Emerging Technologies on City Form and Development, hg. v. Howell, A., H. Tan, A. Brown, M. Schlossberg, J. KarlinResnick, R. Lewis, M. Anderson, N. Larco, G. Tierney, J. Carlton, J. Kim und B. Steckler. Eugene, OR: Urbanism Next Center, University of Oregon, 97-113. https://cpb-us-e1.wpmucdn.com/blogs.uoregon.edu/dist/f/13615/files/2020/01/NSF-Report_All-Chapters_FINAL_013020.pdf (14.5.2020).

Marsden, G., I. Docherty und R. Dowling 2020. „Parking futures: Curbside management in the era of ,new mobility" services in British and Australian cities“, in Land Use Policy 91, 104012. DOI: 10.1016/j. landusepol.2019.05.031.

Mitteregger, M., A. Soteropoulos, J. Bröthaler und F. Dorner 2019. „Shared, Automated, Electric: the Fiscal Effects of the ,Holy Trinity“", Proceedings of the 24. REAL CORP, International Conference on Urban Planning, Regional Development and Information Society, 2.-4.4.2019, Karlsruhe.

Mitteregger, M., E. M. Bruck, A. Soteropoulos, A. Stickler, M. Berger, J. S. Dangschat, R. Scheuvens und I. Banerjee 2020. AVENUE21. Automatisierter und vernetzter Verkehr: Entwicklungen des urbanen Europa. Berlin: Springer Vieweg.

NACTO (National Association of City Transportation Officials) 2017. „Curb Appeal. Curbside Management Strategies for improving transit reliability“. https://nacto.org/wp-content/uploads/2017/11/ NACTO-Curb-Appeal-Curbside-Management.pdf (14.5.2020).

NACTO 2019. „Blueprint for Autonomous Urbanism: Second Edition“. https://nacto.org/publication/ bau2/ (14.5.2020).

Nehrke, G., und W. Loose 2018. „Nutzer und Mobilitätsverhalten in verschiedenen CarSharing-Varianten“, Projektbericht. Berlin: Bundesverband CarSharing e.V. 
orange edge 2016. „Klimaschutzteilkonzept Mobilität. Stadt Königs Wusterhausen: Endbericht“. https:// www.koenigs-wusterhausen.de/817028/KW-Endbericht.pdf (22.10.2020).

Papa, E., und A. Ferreira 2018. „Sustainable Accessibility and the Implementation of Automated Vehicles: Identifying Critical Decisions“, in Urban Science (2) 1, 5.

Ram, M., Jaffri, S., Gerretsen, P., Rigter, D., Chorus, P., und M. Wiers-Faver Linhares 2013. Maak Plaats! Werken aan knooppunt ontwikkeling in Noord-Holland. Rotterdam: Vereniging Deltametropool, provincie Noord-Holland.

Ritter, E.-H. 2017. „Strategieentwicklung heute. Zum integrativen Management konzeptioneller Politik“, in PNDonline 1, 12. http://archiv.planung-neu-denken.de/images/stories/pnd/dokumente/pnd-online_2007-1.pdf (14.5.2020).

Ruchinskaya, T., K. loannidis und K. Kimic 2019. „Revealing the Potential of Public Places: Adding a New Digital Layer to the Existing Thematic Gardens in Thessaloniki Waterfront", in CyberParks - The Interface Between People, Places and Technology: New Approaches and Perspectives, hg. v. C. Smaniotto Costa, I. Šuklje Erjavec, T. Kenna, M. de Lange, K. Ioannidis, G. Maksymiuk und M. de Waal. Cham: Springer International Publishing, 181-195.

SAE International 2018. „Taxonomy and Definitions for Terms Related to Driving Automation Systems for On-Road Motor Vehicles - J3016“, 15.6.2018. www.sae.org/standards/content/j3016_201806/ (20.4.2020).

Schaller, B. 2017. „Empty Seats, Full Streets. Fixing Manhattan's Traffic Problem“. http://schallerconsult. com/rideservices/emptyseats.pdf (14.5.2020).

Schlump, C., T. Wehmeier, B. Helff, G. Reesas, H. Wohltmann, T. Schäfer, A. Kindl und I. Luchmann 2014. „Neue Mobilitätsformen, Mobilitätsstationen und Stadtgestalt“, ExWoSt-Informationen 45/1. Bonn: Bundesinstitut für Bau-, Stadt und Raumforschung im Bundesamt für Bauwesen und Raumordnung. https://www.bbsr.bund.de/BBSR/DE/veroeffentlichungen/exwost/45/exwost45_1.pdf;jsessionid=FAC8F85DD18A6113963D34B4C80F4CAC.live11293?__blob=publicationFile\&v=1 (20.10.2020).

Schmid, C. 2016. „Urbanität und urbane Qualitäten“, in Urbane Qualitäten. Ein Handbuch am Beispie/ der Metropolitanregion Zürich, hg. v. S. Kretz und L. Kueng. Zürich: Edition Hochparterre.

Schnieder, L. 2018. „Netzplanung“, in Betriebsplanung im öffentlichen Personennahverkehr: Ziele, Methoden, Konzepte, hg. v. L. Schnieder. Berlin/Heidelberg: Springer Vieweg, 21-43.

Schwartz, S. 2017. „New Mobility Playbook. Appendix B: Shared Mobility Study Technical Report. Seattle Department of Transportation. https://www.seattle.gov/Documents/Departments/SDOT/NewMobilityProgram/AppendixB.pdf (14.5.2020).

Sinner, M., P. Khaligh und U. Weidmann 2018. „Consequences of automated transport systems as feeder services to rail: SBB fund for research into management in the field of transport. Report", ETH Zürich - Research Collection. DOI: 10.3929/ethz-b-000266025.

Sinner, M., und U. Weidmann 2019. „How does rail perform against autonomous buses? Two case studies in Switzerland“, 19th Swiss Transport Research Conference 2019, Ascona. DOI: 10.3929/ ethz-b-000342826 (15.10.2020).

Soike, R., J. Libbe, M. Konieczek-Woger und E. Plate 2019. „Räumliche Dimensionen der Digitalisierung. Handlungsbedarfe für die Stadtentwicklungsplanung. Ein Thesenpapier“, Difu-Sonderveröffentlichung. https://repository.difu.de/jspui/bitstream/difu/256328/1/DM19101469.pdf (14.5.2020).

Soteropoulos, A., A. Stickler, V. Sodl, M. Berger, J. Dangschat, P. Pfaffenbichler, G. Emberger, E. Frankus, R. Braun, F. Schneider, S. Kaiser, H. Wakolbinger und A. Mayerthaler 2019. „SAFiP - Systemszenarien Automatisiertes Fahren in der Personenmobilität“. Wien: bmvit.

Sousa, N., A. Almeida, J. Coutinho-Rodrigues und E. Natividade-Jesus 2017. „Dawn of autonomous vehicles: Review and challenges ahead“, in Proceedings of the Institution of Civil Engineers - Municipal Engineer (171) 1, 3-14.

Stadt Wien 2018. „Leitfaden Mobilitätsstationen. Die Umsetzung von Mobilitätsstationen in Stadtentwicklungsgebieten am Beispiel Zielgebiet Donaufeld, Wien“, Werkstattberichte der Stadtentwicklung Wien 179. Wien: MA 18 - Stadtentwicklung und Stadtplanung und MA 21 - Stadtteilplanung und Flächennutzung. en.gv.at/stadtentwicklung/studien/b008521.html (15.6.2020).

Stiglic, M., N. Agatz, M. Savelsbergh und M. Gradisar 2015. „The benefits of meeting points in ride-sharing systems“, in Transportation Research Part B: Methodological 82, 36-53.

Taut, B. 1977. Architekturlehre. Grundlagen, Theorie und Kritik, Beziehung zu den anderen Künsten und zur Gesellschaft. VSA, Hamburg 
verkehrsplus 2015. „Ve3 - Planungen von Verknüpfungen an Verkehrsstationen“, ÖBB infra. Download unter (14.5.2020).

Wefering, F., Rupprecht, S., Bührmann, S., und S. Böhler-Baedeker 2013. Guidelines. Developing and Implementing a Sustainable Urban Mobility Plan. Rupprecht Consult - Forschung und Beratung $\mathrm{GmbH}$. http://www.rupprecht-consult.eu/uploads/tx_rupprecht/Revised_SUMP_Guidelines_final_web_Jan_14.pdf (14.5.2020).

Weidmann, U., R. Dorbritz, H. Orth, M. Scherer und P. Spacek 2011. „Einsatzbereiche verschiedener Verkehrsmittel in Agglomerationen“. Schweizerische Eidgenossenschaft: Eidgenössisches Departement für Umwelt, Verkehr, Energie und Kommunikation UVEK. (14.5.2020).

Zhang, W. 2017. „The interaction between land use and transportation in the era of shared autonomous vehicles: A simulation model“, Dissertation, Georgia Institute of Technology, Atlanta, GA. https:// smartech.gatech.edu/bitstream/handle/1853/58665/ZHANG-DISSERTATION-2017.pdf?sequen$\mathrm{ce}=1 \&$ isAllowed=y (14.5.2020).

Zhang, W., S. Guhathakurta, J. Fang, und G. Zhang 2015. „Exploring the impact of shared autonomous vehicles on urban parking demand: An agent-based simulation approach“, in Sustainable Cities and Society 19, 34-45. DOI: 10.1016/j.scs.2015.07.006.

Zhang, W., und K. Wang 2020. „Parking futures: Shared automated vehicles and parking demand reduction trajectories in Atlanta“, in Land Use Policy 91, 103963. DOI: 10.1016/j.landusepol.2019.04.024.

Open Access Dieser Beitrag wird unter der Creative Commons Namensnennung 4.0 International Lizenz (http://creativecommons.org/licenses/by/4.0/deed.de) veröffentlicht, welche die Nutzung, Vervielfältigung, Bearbeitung, Verbreitung und Wiedergabe in jeglichem Medium und Format erlaubt, sofern Sie den/die ursprünglichen Autor(en) und die Quelle ordnungsgemäß nennen, einen Link zur Creative Commons Lizenz beifügen und angeben, ob Änderungen vorgenommen wurden.

Die in diesem Beitrag enthaltenen Bilder und sonstiges Drittmaterial unterliegen ebenfalls der genannten Creative Commons Lizenz, sofern sich aus der Abbildungslegende nichts anderes ergibt. Sofern das betreffende Material nicht unter der genannten Creative Commons Lizenz steht und die betreffende Handlung nicht nach gesetzlichen Vorschriften erlaubt ist, ist für die oben aufgeführten Weiterverwendungen des Materials die Einwilligung des jeweiligen Rechteinhabers einzuholen.

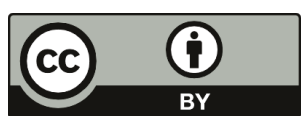

